Published in final edited form as:

Nat Neurosci. 2019 November 01; 22(11): 1793-1805. doi:10.1038/s41593-019-0498-9.

\title{
FUS-mediated regulation of acetylcholine receptor transcription at neuromuscular junctions is compromised in amyotrophic lateral sclerosis
}

\author{
Gina Picchiarelli ${ }^{1, *}$, Maria Demestre ${ }^{2,{ }^{*}}$, Amila Zuko $^{3}$, Marije Been $^{3}$, Julia Higelin $^{2}$, Stéphane \\ Dieterlé $^{1}$, Marc-Antoine Goy ${ }^{1}$, Moushami Mallik ${ }^{3,4,5}$, Chantal Sellier ${ }^{6}$, Jelena Scekic- \\ Zahirovic $^{1}$, Li Zhang ${ }^{4,5}$, Angela Rosenbohm ${ }^{7}$, Céline Sijlmans ${ }^{3}$, Amr Aly ${ }^{2}$, Sina \\ Mersmann $^{4,5}$, Inmaculada Sanjuan-Ruiz ${ }^{1}$, Annemarie Hübers ${ }^{7}$, Nadia Messaddeq ${ }^{6}$, Marina \\ Wagner ${ }^{4,5}$, Nick van Bakel ${ }^{3}$, Anne-Laurence Boutillier ${ }^{8}$, Albert Ludolph ${ }^{7}$, Clotilde Lagier- \\ Tourenne $^{9,10}$, Tobias M. Boeckers ${ }^{2,11, \# \text {, Luc Dupuis }}{ }^{1, \#}$, Erik Storkebaum ${ }^{3,4,5, \#}$
}

${ }^{1}$ Université de Strasbourg, Inserm, UMR-S1118, F-67085, Strasbourg, France ${ }^{2}$ Institute of Anatomy and Cell Biology, Ulm University, $89081 \mathrm{Ulm}$, Germany ${ }^{3}$ Department of Molecular Neurobiology, Donders Institute for Brain, Cognition and Behaviour and Faculty of Science, Radboud University, Nijmegen, Netherlands ${ }^{4}$ Molecular Neurogenetics Laboratory, Max Planck Institute for Molecular Biomedicine, Muenster, Germany ${ }^{5}$ Faculty of Medicine, University of Muenster, Muenster, Germany ${ }^{6}$ IGBMC, INSERM U964, CNRS UMR7104, University of Strasbourg, 67404, Illkirch, France ${ }^{7}$ Department of Neurology, Oberer Eselsberg 45, $89081 \mathrm{Ulm}$ Germany ${ }^{8}$ Université de Strasbourg, Centre National de la Recherche Scientifique, UMR 7364, Laboratoire de Neurosciences Cognitives et Adaptatives, F-67000 Strasbourg, France ${ }^{9}$ Department of Neurology, Massachusetts General Hospital, Harvard Medical School, Charlestown, MA 02129 , USA ${ }^{10}$ Broad Institute of Harvard University and MIT, Cambridge, MA 02142, USA ${ }^{11}$ DZNE, Ulm site, 89081 Ulm, Germany

\section{Abstract}

Users may view, print, copy, and download text and data-mine the content in such documents, for the purposes of academic research, subject always to the full Conditions of use:http://www.nature.com/authors/editorial_policies/license.html\#terms

\# corresponding authors: Erik Storkebaum: e.storkebaum@donders.ru.nl, Phone: +316 257660 73; Luc Dupuis: ldupuis@unistra.fr, Phone: +33 3688534 57; Tobias Boeckers: tobias.boeckers@uni-ulm.de, Phone:+ 49-731-5023220/1.

these authors contributed equally

Reporting Summary

Further information on research design is available in the Life Sciences Reporting Summary linked to this article.

Author contributions

GP performed most of the experiments in heterozygous knock-in mice and in C2C12 cells, with the help of SD, MAG, JSZ and ISR $\mathrm{MD}, \mathrm{JH}$ and AA performed most of the experiments in human derived cells and tissues. AZ, MB, MM, LZ, CS, SM, MW, NvB and ES performed and analyzed the experiments in homozygous knock-in, MyoD-CRE rescued knock-in, and knock-out mice. CS performed immunoprecipitation experiments. NM performed electron microscopy. ALB supervised ChIP experiments. AR provided human muscle biopsies. AH performed and analyzed patient EMG analysis. AL provided patient and clinical material. MD, CLT, TMB, LD and ES initiated, conceived and supervised the project. GP, MD, LD and ES wrote the manuscript. All authors contributed to the experimental design and interpretation and commented on the manuscript.

Competing interest statement

The authors declare no competing interests. 
Neuromuscular junction (NMJ) disruption is an early pathogenic event in amyotrophic lateral sclerosis (ALS). Yet, direct links between NMJ pathways and ALS-associated genes such as FUS, whose heterozygous mutations cause aggressive forms of ALS, remain elusive. In a knock-in FusALS mouse model, we identified postsynaptic NMJ defects in newborn homozygous mutants, attributable to mutant FUS toxicity in skeletal muscle. Adult heterozygous knock-in mice displayed smaller neuromuscular endplates that denervated before motor neuron loss, consistent with 'dying-back' neuronopathy. FUS was enriched in subsynaptic myonuclei, and this innervation-dependent enrichment was distorted in FUS-ALS. Mechanistically, FUS collaborates with the ETS-transcription factor ERM to stimulate transcription of acetylcholine receptor (AchR) genes. FUS-ALS patient iPSC-derived motor neuron-myotube co-cultures revealed endplate maturation defects due to intrinsic FUS toxicity in both motor neurons and myotubes. Thus, FUS regulates AChR gene expression in subsynaptic myonuclei and muscle-intrinsic toxicity of ALSmutant FUS may contribute to dying-back motor neuronopathy.

\section{Introduction}

The neuromuscular junction (NMJ) is the chemical synapse between motor neurons and skeletal muscle fibers. In mammals, neuromuscular transmission is mediated by acetylcholine (ACh) release from presynaptic terminals, which activates the postsynaptic nicotinic acetylcholine receptor (AChR), a ligand-gated ion channel. During development, even before innervation, AChR clusters are already present in the center of the muscle, a process called prepatterning ${ }^{1,2}$. Muscle innervation by motor neurons stabilizes AChR aggregation and results in dispersion of nonsynaptic AChR clusters. Interestingly, upon innervation, transcription of $\mathrm{AChR}$ subunit (Chrn) genes becomes confined to myonuclei underlying the neuromuscular contact, referred to as 'subsynaptic' nuclei ${ }^{2,3}$. This process is mediated by Agrin, which is secreted from motor nerve terminals and binds to its postsynaptic receptor Lrp4, leading to activation of MuSK and the downstream Rac/ MKK7/JNK pathway ${ }^{2,3}$. This results in activation of the ETS transcription factor ERM, also known as ETV5, which binds to N-box elements in the promoters of synapse-specific genes, including Chrn genes ${ }^{4}$, and is associated with specific chromatin modifications in subsynaptic nuclei ${ }^{5}$. In addition to the agrin-LRP4-MuSK-ERM axis, activation of ErbB receptors by extracellular neuregulin may have modulatory roles in MuSK-regulated synaptic gene expression ${ }^{2,3}$.

Structural and functional NMJ defects have been implicated in a plethora of diseases ${ }^{2}$, including the motor neurodegenerative disease amyotrophic lateral sclerosis (ALS) ${ }^{6}$. ALS is considered a 'dying-back' motor neuronopathy, as at least in SOD1-ALS mouse models and a sporadic ALS (sALS) patient, denervation of neuromuscular endplates precedes axonal degeneration and loss of motor neuron cell bodies ${ }^{7-9}$. The most aggressive forms of ALS, with early onset and rapid disease progression, are caused by heterozygous mutations in $F U S$, which encodes a DNA/RNA binding protein involved in several steps of gene expression regulation, including regulation of transcription, chromatin remodeling, mRNA splicing and mRNA subcellular localization ${ }^{10}$. Along with FUS, additional RNA-binding proteins are linked to ALS, including TDP-43, TAF15, EWSR1, hnRNPA2B1, hnRNPA1 and Matrin-3, thus implicating defects in RNA biogenesis in ALS pathogenesis ${ }^{6,11}$. Beyond 
ALS, cytoplasmic inclusions containing FUS or TDP-43 are pathological hallmarks in respectively $\sim 10 \%$ and $\sim 45 \%$ of patients with frontotemporal dementia, the most common form of dementia before the age of $60^{11}$. The vast majority of ALS-causing FUS mutations cluster in the extreme C-terminus of FUS, either missense mutations in the nuclear localization signal (NLS) or frameshift or nonsense (truncating) mutations in or before the NLS $^{12}$. These mutations result in cytoplasmic FUS mislocalization and concomitant reduction of nuclear FUS levels, what is likely an important event in ALS pathogenesis ${ }^{11,13}$.

We previously generated a knock-in Fus-ALS mouse model, which expresses a truncated FUS protein that lacks the $20 \mathrm{C}$-terminal amino acids which constitute the NLS (Fus ${ }^{\Delta N L S}$ mice $)^{14}$. Reminiscent of FUS-ALS, in motor neurons the Fus ${ }^{\Delta N L S}$ mutation results in FUS cytoplasmic mislocalization and reduced nuclear FUS levels. To discriminate between phenotypes induced by loss of nuclear FUS function versus toxicity induced by cytoplasmic FUS mislocalization, we generated Fus knockout mice $\left(\mathrm{Fus}^{-/-} \text {mice }\right)^{14}$. Overall, homozygous $\mathrm{FuS}{ }^{\mathrm{N} L S / \triangle N L S}$ and $\mathrm{Fus}^{-/-}$mice displayed similar phenotypes, most prominently perinatal lethality due to respiratory insufficiency, likely attributable to loss of FUS function. However, loss of $\sim 30 \%$ of motor neuron cell bodies was found in Fus ${ }^{4 N L S / \triangle N L S}$ but not $\mathrm{FuS}^{-/-}$mice, suggesting an additional gain-of-toxic-function mechanism ${ }^{14}$. Heterozygous Fus ${ }^{\Delta N L S /+}$ mice provide a mouse model for FUS-ALS, as they recapitulate several pathological hallmarks and display adult-onset progressive motor neurodegeneration associated with motor deficits ${ }^{15}$.

Here, we investigated the possible contribution of NMJ defects to FUS-ALS. Fus mutant mouse models displayed predominantly postsynaptic defects: the endplate surface area and the total number of endplates in hind limb muscles were significantly reduced in newborn Fus ${ }^{\Delta N L S / \triangle N L S}$ mice. Adult Fus $S^{\Delta N L S /+}$ mice displayed reduced endplate surface area and progressive endplate denervation preceding motor neuron loss. Selective reversal of the

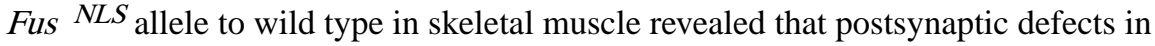
$F u S^{\triangle N L S / \triangle N L S}$ mice are attributable to intrinsic toxicity of the cytoplasmically mislocalized FUS protein in muscle. Furthermore, FUS was enriched in subsynaptic nuclei and stimulates transcription of Chrn genes in collaboration with ERM. These findings may be relevant for human FUS-ALS, as studies in induced pluripotent stem cell (iPSC)-derived myotubes and motor neurons of FUS-ALS patients demonstrated intrinsic toxicity of ALS-mutant FUS in both motor neurons and myotubes. In addition, electromyography (EMG) and muscle biopsies from FUS-ALS patients revealed myogenic pathological activity and impaired enrichment of FUS in subsynaptic nuclei. Together, our data indicate that FUS regulates the synapse-specific expression of Chrn genes, that NMJ pathology is likely an early event in FUS-ALS, and that ALS-mutant FUS is not only toxic in motor neurons but also in muscle.

\section{Results}

\section{Neuromuscular junction morphology defects in newborn Fus mutant mice}

As $\mathrm{Fus}^{-1}$ and $\mathrm{Fus} \mathrm{S}^{4 N L S / \Delta N L S}$ mice die shortly after birth ${ }^{14}$, we evaluated NMJ morphology in newborn mice. Surprisingly, in the tibialis anterior (TA), endplate innervation was not different between Fus ${ }^{\triangle N L S / \Delta N L S}$ and littermate controls (Figure 1a,b,e). However, endplate surface area was reduced in Fus $\triangle N L S / \triangle N L S$ muscles, by $\sim 27 \%$ as compared to control (Figure 
1f), and the total number of endplates in Fus ${ }^{\Delta N L S / \triangle N L S}$ TA was reduced ( 19\%, Figure 1g). In $\mathrm{Fus}^{-/-}$muscles, endplates were normally innervated (Figure 1c,d,h), and endplate area was not altered (Figure 1i). However, similar to Fus $\Delta N L S / \Delta N L S$, the total number of endplates in the TA was reduced by $\sim 25 \%$ (Figure $1 \mathrm{j}$ ). Similarly, in the gastrocnemius the innervation status was not changed in $\mathrm{Fu} S^{\triangle N L S / \triangle N L S}$ or $\mathrm{Fus}^{-/-}$, endplate area was reduced in Fus ${ }^{\Delta N L S / \triangle N L S}(\sim 17 \%)$, but not Fus ${ }^{-/-}$, and the total number of endplates was reduced in both Fus ${ }^{\Delta N L S / \triangle N L S}(\sim 33 \%)$ and Fus $s^{-/}(\sim 44 \%)$ (Supplementary Figure 1a-f). Together, these findings point towards a postsynaptic defect in Fus mutant NMJs.

Ultrastructural analysis of E18.5 gastrocnemius revealed morphological defects in Fus mutant NMJs that appeared slightly more pronounced in Fus $\Delta N L S / \Delta N L S$ than in $\mathrm{Fus}^{-/-}$mice (Figure 1k-n). This was confirmed by semi-quantitative analysis (Supplementary Figure 1gk). Pre- and postjunctional membranes were frequently not apposed in Fus mutant mice (Supplementary Figure 1g) and the characteristic invaginations of the postsynaptic muscle membrane (postjunctional folds) were often missing (Supplementary Figure 1h). In addition, the continuity of both the neuronal and muscle membrane was frequently disrupted (Supplementary Figure 1i,j), presynaptic terminals often lacked visible synaptic vesicles and mitochondria (Figure 11, Supplementary Figure 1k), and some Fus ${ }^{\Delta N L S / \Delta N L S}$ NMJs showed signs of presynaptic degeneration (Figure 11).

As a reduced number of muscle fibers may explain the reduced endplate number in FUS mutant muscles, we quantified the total number of muscle fibers in the extensor digitorum longus (EDL) and TA muscles of mutant Fus and control newborn pups (Supplementary Figure 2a-e). Quantification revealed no significant differences in the total number of muscle fibers in Fus $\triangle N L S / \triangle N L S$ or Fus ${ }^{-/-}$EDL (Supplementary Figure 2c,d), or Fus ${ }^{-/-}$TA (Supplementary Figure 2e). Thus, it is unlikely that the reduced endplate number in FUS mutant muscles is attributable to a reduced number of muscle fibers.

\section{Cytoplasmic mislocalized Fus ${ }^{\triangle \mathrm{NLS}}$ protein is intrinsically toxic to muscle}

Given the predominantly postsynaptic defects in Fus $\Delta N L S / \Delta N L S$ and Fus ${ }^{-/-}$NMJs, we evaluated muscle-intrinsic toxicity of FUS ${ }^{\triangle N L S}$ protein, by selectively reverting the Fus $\Delta N L S$ allele to wild type in skeletal muscle. $M y o D^{i C r e}$ mice were selected for this purpose, as they selectively express iCre in committed myogenic progenitor cells from E10.5 onwards ${ }^{16,17}$. Quantification of recombination efficiency in $R O S A^{m T / m G}$ reporter mice ${ }^{18}$ confirmed that $M y o D^{i C r e}$ mediates highly efficient ( $>99.5 \%$ ) recombination in skeletal muscle fibers (Supplementary Figure 3).

While in Fus ${ }^{\triangle N L S / \triangle N L S}$ muscle, FUS displayed a diffuse cytoplasmic localization and was no longer enriched in nuclei, muscle-selective reversal of the Fus $\Delta N L S$ allele restored FUS nuclear localization (Figure 2a). Quantitative analysis of cytoplasmic:nuclear FUS localization confirmed these observations (Figure 2b). To assess whether selective restoration of FUS subcellular localization in muscle fibers rescues NMJ morphology, we quantified endplate surface area in the TA. As expected, endplate area was reduced in $M y o D$ ${ }_{+{ }_{+}^{+} ; F u s}^{\Delta N L S / \triangle N L S}$ mice (Figure 2c). Strikingly, in $M y o D^{i C r e /+} ; F u s^{\Delta N L S / \Delta N L S}$ mice, endplate

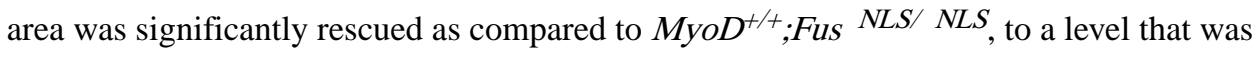
not significantly different from control genotypes (Figure 2c). These data are consistent with 
cell-autonomous toxicity of FUS ${ }^{\Delta N L S}$ protein in skeletal muscle. Of note, in spite of rescuing postsynaptic defects, the presence of $M y o D^{i C r e}$ did not rescue the early postnatal lethality of Fus ${ }^{\triangle N L S / \triangle N L S}$ mice, indicating that FUS ${ }^{\Delta N L S}$ toxicity in other cell types is sufficient to cause neonatal lethality.

\section{Neuromuscular junction defects in adult heterozygous Fus $^{\triangle N L S /+}$ mice}

Whereas homozygous Fus ${ }^{\Delta N L S / \Delta N L S}$ and $\mathrm{Fus}^{-/-}$mice die shortly after birth ${ }^{14}$, heterozygous Fus ${ }^{\Delta N L S /+}$ mice develop adult-onset motor neurodegeneration after 10 months of age with associated motor deficits ${ }^{15}$. Evaluation of NMJ morphology in Fus ${ }^{\Delta N L S /+}$ TA revealed significant endplate denervation already at 1 month, which progressively worsened with age (Figure 3a-d). Endplate surface area was significantly reduced in Fus ${ }^{\Delta N L S /+}$ at both 1 and 10 months (Figure 3f,g), while the total number of endplates in the TA of 1-month-old Fus ${ }^{\Delta N L S /+}$ mice was not altered (Supplementary Figure 2f). Consistent with the reduced endplate area which presumably corresponds to a reduced $\mathrm{AChR}$ number, repetitive stimulation of the sciatic nerve at $20 \mathrm{~Hz}$ and $50 \mathrm{~Hz}$ resulted in a successive decrease in compound muscle action potential (CMAP) amplitude between first and tenth stimulus in Fus ${ }^{\Delta N L S /+}$ but not Fus ${ }^{+/+}$mice (Figure 3i), indicating that the 'safety factor' of neuromuscular transmission is reduced in $F u s^{\Delta N L S /+}$ mice. Together, these data show chronic endplate denervation, progressing with age, accompanied with an early and sustained reduction of endplate size in Fus ${ }^{\Delta N L S /+}$ mice. Importantly, in 28-month-old heterozygous $\mathrm{Fus}^{+/-}$mice, endplate innervation status and surface area was not significantly different from control (Figure 3e,h). Thus, heterozygous loss of Fus function is not sufficient, and gain-of-toxic function is required to induce NMJ defects in Fus ${ }^{\Delta N L S /+}$ mice.

\section{FUS is enriched in subsynaptic nuclei dependent on innervation}

Our findings suggested that FUS may regulate expression of Chrn genes, which become exclusively expressed in subsynaptic nuclei upon innervation ${ }^{2,3}$. This prompted us to investigate FUS subcellular localization in adult gastrocnemius, in relation to endplate localization. Intriguingly, FUS was significantly enriched in subsynaptic nuclei, defined as nuclei located directly underneath endplates. (Figure 4a,b). In contrast, FUS subsynaptic enrichment was lost in 10-month-old Fus ${ }^{\Delta N L S /+}$ mice (Figure 4a,b).

Interestingly, FUS subsynaptic enrichment was dependent on innervation, as unilateral axotomy of the sciatic nerve in adult wild type mice revealed that 5 days after injury, FUS subsynaptic enrichment was lost in ipsilateral as compared to contralateral muscles (Figure $4 c, d)$. Thus, FUS subsynaptic enrichment is dependent on innervation and disrupted in the presence of FUS ${ }^{\triangle N L S}$ protein.

\section{FUS collaborates with ERM to regulate Chrn gene expression}

To evaluate whether FUS regulates expression of Chrn genes, $\mathrm{C} 2 \mathrm{C} 12$ mouse myoblast cells were treated with siRNA targeting FUS (siFus) or control siRNA (siCt), and differentiated for 2 days. siFus induced strong FUS knock-down, both at the transcript (Figure 5a) and the protein level (Figure 5b). Interestingly, FUS knock-down reduced expression of each of the five Chrn genes by $>50 \%$ (Figure 5c). Consistently, transcript levels of several AChR 
subunits were significantly reduced in muscles of E18.5 Fus ${ }^{\Delta N L S / \Delta N L S}$ and 1-month-old Fus ${ }^{\Delta N L S /+}$ mice (Supplementary Figure 4a,b).

Chromatin immunoprecipitation in $\mathrm{C} 2 \mathrm{C} 12$ cells using two different anti-FUS antibodies revealed that FUS binds the promoters of Chrnal, Chrnd and Chrne genes (encoding the AChR alpha1, delta and epsilon subunits, respectively), but not the histone 2ac (H2ac) promoter. Importantly, FUS binding at Chrn promoters was similar to its binding at the Mecp 2 promoter, a known FUS target ${ }^{19}$ (Figure 5d). These data are consistent with the presence of predicted FUS binding sites in the promoter regions of murine and human $C H R N$ genes (Supplementary Note 1). To evaluate a direct promoter-dependent transcriptional mechanism, $\mathrm{C} 2 \mathrm{C} 12$ cells were transfected with reporter constructs in which the Chrnd or Chrne promoters drive luciferase expression ${ }^{5}$. For both promoters, but not the $C M V$ promoter, FUS knock-down reduced luciferase expression (Figure 5e-g). Conversely, overexpression of wild type human FUS induced Chrnd and Chrne promoter activity (Figure 5h). Thus, FUS induces transcription of Chrnd and Chrne genes by a promoter-dependent mechanism. Importantly, overexpression of ALS-mutant FUS_R495X did not induce Chrnd or Chrne promoter activity (Figure 5h). This correlated with subcellular mislocalization of FUS_R495X, which displayed cytoplasmic localization, in contrast to nuclear localization of wild type FUS (Supplementary Figure 5). In addition, when co-expressed with wild type FUS, FUS_R495X dose-dependently inhibited the induction of Chrne promoter activity by wild type FUS (Figure 5i), suggesting that FUS_R495X exerts a dominant negative effect on FUS-induced Chrne promoter activity. Co-transfection of $\mathrm{C} 2 \mathrm{C} 12$ cells with differentially tagged wild type and R495X FUS indicated that this dominant negative effect may be attributable to (i) increased localization of wild type FUS to the cytoplasm in the presence of FUS_R495X, and (ii) a more granular localization pattern of wild type FUS in the nucleus, together resulting in reduced levels of diffusely distributed wild type FUS in the nucleus (Supplementary Figure 5).

Treatment of $\mathrm{C} 2 \mathrm{C} 12$ cells with agrin induced Chrne promoter-driven luciferase expression (Supplementary Figure 6a). Strikingly, knock-down of FUS prevented induction of Chrneluciferase expression by agrin, indicating that FUS is required for agrin-induced Chrne transcription (Supplementary Figure 6a). Selective Chrne expression in subsynaptic nuclei is mediated by the ETS transcription factor ERM ${ }^{4}$. Importantly, ERM knock-down largely decreased FUS-induced Chrne promoter activity (Figure 5j), while conversely, activation of Chrne promoter activity by ERM overexpression was severely blunted by FUS knock-down (Figure 5k). Finally, immunoprecipitation in HEK293 cells transfected with differentially tagged FUS and ERM revealed that both proteins interact (Supplementary Figure 6b), and such interaction was also detected using immunoprecipitation of endogenous proteins in mouse (C2C12) and human (HSkMC) myoblast cell lines (Figure 51,m), as well as in HEK293 cells (Supplementary Figure 6c). Importantly, the FUS-ERM interaction was not affected by RNase treatment (Figure 5l,m; Supplementary Figure 6b,c), and therefore RNAindependent. Altogether, our results indicate that subsynaptic gene expression is mediated by the synergistic action of FUS and ERM on AChR-encoding gene promoters. 


\section{Impaired endplate maturation in iPSC-derived myotubes from FUS-ALS patients}

To explore the potential relevance of our findings in mutant Fus mouse models for human FUS-ALS, we studied endplate maturation and NMJ formation in cultured myotubes and motor neuron-myotube co-cultures derived from FUS-ALS patients and neurologically healthy controls. iPSC lines derived from juvenile ALS patients carrying R495QfsX527 (FUS1) or D502Tfs*27 (FUS2) frameshift mutations ${ }^{20}$, two control lines (CNTL1 and CNTL2), and an isogenic control line (CNTL3) obtained by correcting the FUS1 mutation by CRISPR/Cas9 technology ${ }^{20}$ were used for these experiments (Supplementary Table 1).

As a first paradigm to test the effect of ALS-mutant FUS on AChR clustering and endplate maturation, patient and control iPSCs were differentiated in myotubes (Supplementary Figure 7a $)^{21}$. Extensive characterization of expression levels and localization of myogenic markers ( $P A X 7, M Y O G$, MHC, desmin, a-actinin, RyR1 and DHPRa) confirmed appropriate myogenic proliferation and differentiation in mature myotubes of both FUSALS and control lines (Supplementary Figure 7b-d). Surprisingly, FUS predominantly localized to the nucleus in both control and FUS-ALS myotubes (Supplementary Figure 7d), in contrast to cytoplasmic mislocalization of ALS-mutant FUS in motor neurons ${ }^{20}$.

To characterize endplate morphology, endplates were categorized in three groups based on AChR clustering: (i) diffuse puncta, (ii) aligned puncta, and (iii) dense cluster (Figure 6a), reflecting subsequent steps in endplate maturation. Quantitative analysis revealed impaired endplate maturation in FUS-ALS myotubes as compared to control (Figure 6b).

Finally, expression of $C H R N$ genes was evaluated at different time points during myoblast proliferation and myotube differentiation. While transcript levels of the AChR gamma and epsilon subunits were not significantly altered, AChR alpha1 transcript levels were substantially reduced in FUS-ALS myotubes (Supplementary Figure 7b), consistent with the endplate maturation defect and the data in mutant Fus mouse models and $\mathrm{C} 2 \mathrm{C} 12$ cells.

\section{ALS-mutant FUS is intrinsically toxic to motor neurons and myotubes}

To evaluate the effect of motor innervation on endplate maturation, we co-cultured ${ }^{22}$ wild type (CNTL1) or FUS-ALS (FUS1 or FUS2) motor neurons and myotubes for three weeks in all possible CNTL/FUS combinations. Endplate maturation was evaluated as in myotube monocultures, but a fourth category of 'pretzel'-shaped endplates (most mature) was added (Figure 6c).

Consistent with the endplate maturation defect in myotube monocultures, FUS-ALS myotubes co-cultured with CNTL1 motor neurons displayed significantly less mature endplates as compared to CNTL1-CNTL1 co-cultures (Figure 6d). Co-culturing FUS1 motor neurons and myotubes significantly aggravated the endplate maturation defect (Figure 6d), suggesting that mutant motor neurons have a detrimental effect on endplate maturation.

Consistently, co-culturing of FUS-ALS motor neurons with CNTL myotubes was sufficient to impair endplate maturation (Figure 6d). Measurement of single puncta size within endplates revealed that, as compared to CNTL1-CNTL1 co-cultures, combinations containing FUS-ALS motor neurons and/or myotubes displayed significantly reduced single 
particle size (Supplementary Figure 8a), confirming defective AChR clustering in the presence of FUS-ALS cells.

To exclude potential confounding genetic background effects, we performed motor neuronmyotube co-cultures derived from FUS1 and its isogenic CNTL3. This experiment revealed an even more striking endplate maturation defect in co-cultures in which either myotubes or motor neurons carried the FUS_R495QfsX527 mutation, and the maturation defect was most severe when both myotubes and motor neurons were FUS mutant (Figure 6e,

Supplementary Figure 8b).

Immunostaining revealed that in CNTL-CNTL motor neurons-myotube cultures, FUS localized to nuclei in motor neurons and myotubes. In cultures of CNTL myotubes and FUSALS motor neurons, FUS localized to both nucleus and cytoplasm in motor neurons, whereas FUS predominantly localized to nuclei in myotubes, even in co-cultures of FUS mutant motor neurons and myotubes (Supplementary Figure 8c).

Expression analysis of myogenic differentiation markers revealed that in FUS2 'full' mutant co-cultures, $M Y O D$ and $M Y O G$ transcript levels were significantly reduced, suggesting impaired myogenic differentiation (Supplementary Figure 8d). Furthermore, $M Y O G$ expression levels were also significantly reduced when either motor neurons or myotubes were FUS2 mutant. In addition, transcript levels of AChR alpha 1 and gamma subunits were substantially reduced in FUS2 full mutant co-cultures, while AChR epsilon transcript levels were not changed (Supplementary Figure 8d). These data are consistent with the most pronounced endplate differentiation defect in FUS-ALS full mutant co-cultures.

\section{Muscle biopsies from FUS-ALS patients show muscle atrophy, endplate denervation, FUS mislocalization and loss of FUS enrichment in subsynaptic nuclei}

To further investigate the potential clinical relevance of our findings, we analyzed muscle biopsies from three controls and three FUS-ALS patients with R495QfsX527 (FUS1, same patient as FUS1 iPSC line) and K510R (FUS4 and FUS5) mutations (Supplementary Table 2). EMG surprisingly revealed myopathic changes in FUS-ALS patients. Specifically, small and polyphasic motor unit action potentials (MUAPs) were detected in FUS1 and FUS5 (see Methods for details). In FUS1, myopathic changes were seen alongside neurogenic changes, but in FUS5, only myopathic changes were found. All patients showed pathological spontaneous activity (PSA) in the body regions studied, according to the electrophysiological Awaji Criteria ${ }^{23}$ (Supplementary Table 2). H\&E staining on muscle biopsy sections revealed neurogenic muscle atrophy in the three FUS-ALS patients, with groups of small angulated fibers, increased density of muscle nuclei and increased presence of connective tissue, in particular in patients FUS4 and FUS5 (Figure 7a). Quantitative analysis confirmed the abundant presence of very small muscle fibers (surface area $<500 \mu \mathrm{m}^{2}$ ) in each of the FUS-ALS patients, never found in control biopsies (Figure $7 \mathrm{~b}$ and Supplementary Figure 9a). Furthermore, muscle fiber size was highly heterogeneous in FUS1, with a small fraction of abnormally large fibers. The average muscle fiber surface area was significantly reduced in FUS1 (compared to CNTL4 and CNTL5), FUS4 and FUS5 (compared to all controls) (Figure 7b), and the density of nuclei was increased in FUS4 and FUS5 (Supplementary Figure 9b). Immunostaining revealed endplate denervation in FUS- 
ALS muscle biopsies (Figure 7c). Endplate surface area in FUS-ALS biopsies was not significantly different from CNTL4 and CNTL5, but significantly reduced as compared to CNTL6 (Supplementary Figure 9d). In FUS-ALS biopsies, nuclear FUS staining often displayed a granular pattern, in contrast to diffuse staining in controls (white arrowheads in Figure 7c), and cytoplasmic FUS mislocalization was occasionally observed in FUS-ALS biopsies (red arrowheads). Quantification of FUS staining intensity showed that on average, FUS nuclear staining was not significantly changed in FUS-ALS biopsies, and cytoplasmic FUS staining was increased in FUS5 but not FUS1 and FUS4 (Supplementary Figure 9c). However, importantly, FUS was enriched in subsynaptic nuclei in control biopsies by about 2 -fold, but this enrichment was completely lost in FUS1 and substantially reduced in FUS4 (Figure 7d). Thus, similar to muscles of Fus ${ }^{\Delta N L S /+}$ mice, FUS subsynaptic enrichment is affected in FUS-ALS patients.

\section{Discussion}

Motor neuron degeneration (MND) in ALS is thought to occur in a retrograde fashion: NMJs are affected first, followed by motor axonal degeneration and finally degeneration of motor neuron cell bodies. This 'dying-back' pattern of MND was reported in SOD1-ALS mouse models ${ }^{7-9}$ and a single sALS patient ${ }^{8}$, but the potential broader relevance for other familial ALS forms, and the broad sALS population is unclear. Here, we identified NMJ morphology defects in FUS-ALS mouse models carrying a Fus mutation that results in deletion of the last 20 amino acids of the endogenous FUS protein, akin human FUS-ALS mutations ${ }^{12}$ and affecting the NLS and thus impairing FUS nuclear import ${ }^{13}$.

Neuromuscular endplate surface area was reduced in newborn Fus $\triangle N L S / \Delta N L S$ pups and adult $\mathrm{FuS}^{\mathrm{ANLS} /+}$ mice, but not in $\mathrm{FuS}^{-/}$pups or Fus ${ }^{+/}$mice, suggesting an underlying gain-oftoxic-function mechanism. The total number of endplates in entire hind limb muscles was reduced in both Fus ${ }^{\Delta N L S / \triangle N L S}$ and $\mathrm{Fus}^{-/-}$pups, suggesting an additional loss-of-function mechanism. Consistently, iPSC-derived myotube cultures and motor neuron-myotube cocultures from $F U S$-ALS patients displayed impaired endplate maturation, underscoring the potential relevance of our findings in mouse models for human FUS-ALS. Importantly, in adult Fus ${ }^{\Delta N L S /+}$ mice, in which motor neuron loss only occurs after the age of 10 months ${ }^{15}$, endplate denervation was already detected at one month of age, and progressively worsened. Thus, MND occurs in a dying-back pattern in this FUS-ALS mouse model, consistent with recent observations in transgenic mice overexpressing wild type or ALS-mutant human FUS $^{24,25}$, and in human iPSC-derived FUS-ALS motor neuron models ${ }^{26}$.

An unresolved question is whether skeletal muscle fibers are merely bystanders or rather actively contribute to MND in ALS. This conundrum was investigated in SOD1-ALS mouse models, with conflicting results. Indeed, two studies reported that reducing mutant SOD1 expression in skeletal muscle of SOD1-ALS mice does not affect disease course, neither did increasing muscle fiber number and diameter by follistatin expression ${ }^{27,28}$. On the other hand, selective expression of ALS-mutant SOD1_G93A in skeletal muscle resulted in progressive muscle atrophy, reduced muscle strength, alteration in the contractile apparatus and mitochondrial dysfunction ${ }^{29}$. Strikingly, another study reported that skeletal musclerestricted expression of wild type, G37R and G93A SOD1 resulted in limb weakness, paresis and motor defects, associated with not only severe skeletal muscle pathology, but 
also marked NMJ abnormalities and spinal motor neuron pathology ${ }^{30}$. Furthermore, loss of skeletal muscle-specific microRNA miR-206 accelerates NMJ denervation and disease progression in SOD1 G93A mice ${ }^{31}$. In this study, we show that muscle-selective reversal of the Fus ${ }^{\Delta N L S}$ allele to wild type restored FUS subcellular localization (from cytoplasmic mislocalization to appropriate nuclear localization) and rescued the reduced endplate surface area in $F u s^{\Delta N L S / \Delta N L S}$ newborn pups, showing that this postsynaptic defect is attributable to muscle-intrinsic toxicity of FUS ${ }^{\Delta \mathrm{NLS}}$ protein. Consistently, myotubes derived from FUSALS patients displayed impaired endplate maturation, even when co-cultured with control motor neurons. These findings call for future research to investigate a possible non-cellautonomous contribution of skeletal muscle to the dying-back neuronopathy in FUS-ALS.

Besides toxicity in skeletal muscle, intrinsic toxicity of mutant FUS in motor neurons is likely a key event in triggering MND in FUS-ALS. Indeed, ultrastructural analysis in Fus ${ }^{\triangle N L S / \triangle N L S}$ muscles (this study) and in transgenic mice overexpressing wild type or P525L mutant FUS revealed presynaptic defects ${ }^{24,25}$. Furthermore, selective overexpression of FUS_P525L in motor neurons was sufficient to induce progressive motor neuron loss and NMJ denervation ${ }^{25}$, and motor neuron-selective reversal of the Fus ${ }^{\Delta N L S}$ allele in both $F u S^{\Delta N L S / \Delta N L S}$ and $F u S^{\Delta N L S /+}$ mice prevented progressive motor neuron loss ${ }^{14,15}$, indicating that expression of ALS-mutant FUS in motor neurons is both necessary and sufficient to induce motor neuron loss. Similarly, in motor neuron-myotube co-cultures, FUS-ALS motor neurons induced defective endplate maturation, even when co-cultured with control myotubes. Intriguingly, however, in spite of preventing motor neuron loss, ChAT-cre delayed, but did not prevent development of motor deficits in Fus ${ }^{\Delta N L S /+}$ mice ${ }^{15}$, suggesting the involvement of cell types other than motor neurons. Our finding that toxicity of FUS $^{\triangle N L S}$ protein in myotubes induces NMJ defects suggests that skeletal muscle may contribute to motor deficits in $F u s^{4 N L S /+}$ mice, although this remains to be experimentally proven.

The reduced endplate size and number, and electrophysiological findings in Fus-ALS mouse models prompted us to investigate a possible role of FUS in regulation of Chrn gene expression. Indeed, downregulation of subsynaptic gene expression in Erm mutant mice was associated with reduced endplate surface area $^{4}$. Consistent with a role for FUS in Chrn gene regulation, Chrn transcript levels were reduced in (i) $\mathrm{C} 2 \mathrm{C} 12$ mouse myoblast cells upon FUS knock-down, (ii) muscle of Fus ${ }^{\Delta N L S / \triangle N L S}$ and Fus ${ }^{\Delta N L S /+}$ mice, and (iii) FUS-ALS myotube monocultures and motor neuron-myotube co-cultures.

As expression of AChR subunits is restricted to subsynaptic nuclei in innervated muscles, mediated by agrin-LRP4-MuSK-ERM signaling ${ }^{1,2}$, and the transcription factor ERM is highly enriched in subsynaptic nuclei ${ }^{4}$, we evaluated potential enrichment of FUS in subsynaptic nuclei. Consistent with a role for FUS in stimulating synaptic gene expression, FUS was enriched in subsynaptic nuclei, and this enrichment was lost upon denervation and in Fus ${ }^{\Delta N L S /+}$ mice (independent of denervation).

Experiments in $\mathrm{C} 2 \mathrm{C} 12$ cells revealed that FUS stimulates transcription of Chrn genes. Importantly, FUS was required for induction of Chrne promoter activity by neural agrin, suggesting that FUS is required for ERM function. Consistently, ChIP experiments showed 
that FUS binds $C h r n$ promoters, and co-IP showed that FUS and ERM proteins interact in an RNA-independent manner. Last, FUS-mediated induction of Chrne promoter activity was ERM-dependent, and ERM-mediated induction of the Chrne promoter was FUS-dependent. Of note, ERM was recently reported to directly interacts with $\mathrm{EWS}^{32}$, a protein which, together with FUS and TAF-15, constitutes the FET protein family ${ }^{10}$. The domains in FUS and EWS that mediate ERM interaction remain to be identified. Together, these findings suggest that FET proteins may be critical regulators of multiple ETS transcription factor activities. Besides NMJ development and maintenance, the synergistic action of ETS transcription factors and FET proteins may play a role in development of malignant diseases $^{32}$. Taken together, our data suggest a model in which agrin-Lrp4-MuSK signaling results in recruitment of FUS and ERM to promoter regions of subsynaptic genes, increasing their expression (Supplementary Figure 10).

To the best of our knowledge, this is the first time that an ALS gene is directly implicated in NMJ maintenance and stability. Given the fact that NMJ pathology is considered an early event in ALS pathogenesis, this may constitute an important novel insight. Furthermore, a possible contribution of FUS to the pathogenesis of other neuromuscular diseases with prominent NMJ pathology, including myasthenic syndromes, peripheral neuropathy and spinal muscular atrophy is conceivable. Our data further indicate that ALS-mutant FUS is intrinsically toxic to both motor neurons and skeletal muscle, implying that therapeutic approaches for FUS-ALS - and possibly all forms of ALS - should ideally target both cell types. In conclusion, we discovered that FUS and ERM collaborate to promote the transcription of Chrn genes in subsynaptic nuclei. Impairment of this novel FUS function in FUS-ALS mouse and cell models suggests that possibly, intrinsic toxicity of mutant FUS in skeletal muscle may contribute to the dying-back motor neuronopathy in FUS-ALS patients.

\section{Methods}

\section{Compliance with ethical standards}

Mouse experiments were approved by the local ethical committee from Strasbourg University (CREMEAS) under reference numbers 2015070711529814 and 2016021114497517, and by the LANUV-NRW under reference numbers 84-02.04.2011.A100 and 84-02.04.2016.A166. Human hair and skeletal muscle biopsy donors gave informed consent for the study prior to sampling. All studies with human material were approved by the ethical committee of Ulm University (Nr. 0148/2009 and 265/12 for hairs and 12/09 for muscle biopsies) and in compliance with the guidelines of the Federal Government of Germany (Nr. O.103). Experiments were in accordance with the Declaration of Helsinki concerning Principles for Medical Research containing Human Subjects.

\section{Animals}

Transgenic mice were housed in the Faculty of medicine from Strasbourg University and in the animal facility of the Max Planck Institute for Molecular Biomedicine, with 12/12 h of light/dark cycle and unrestricted access to food and water. 
Fus knock-in, Fus knock-out mice and $M y o D^{i C r e}$ were previously described ${ }^{14,15,16,17}$. Fus knock-in mice express a truncated FUS protein that lacks the PY-NLS, which is encoded by exon 15 of the Fus gene. This mutation can be reverted to a wild type FUS protein upon CRE-mediated recombination ${ }^{14,15}$. Mice heterozygous and homozygous for the targeted allele are referred to as Fus ${ }^{\Delta N L S /+}$ and Fus $\Delta N L S / \Delta N L S$, respectively. The genetic background of all mice used in this study is C57B16/J. Both male and female mice were used in all experiments, except for Figure 3e,h,i, where exclusively females were used, and Figure 3c,d,f,g, Figure 4b,d, Supplementary Figure $2 \mathrm{f}$ and Supplementary Figure 4b, where exclusively males were used.

\section{Histology, immunohistochemistry and morphometric analysis}

Information on antibodies is summarized in Supplementary Table 3.

NMJ morphology in newborn and 1-month-old mice-After sacrifice and removal of the skin, the bodies of the pups were fixed in $4 \%$ paraformaldehyde (PFA) in PBS on ice, washed in PBS and stored at $4{ }^{\circ} \mathrm{C}$. After genotyping, gastrocnemius and TA muscles were embedded in 3\% agarose in PBS and cut in $100 \mu \mathrm{m}$ longitudinal sections using vibratome.

Free floating sections were permeabilized for $30 \mathrm{~min}$ in PTT (PBS + 0.5\% Triton X-100, $0.1 \%$ Tween 20), washed in PBS (3x $15 \mathrm{~min}$ ) and blocked for $1 \mathrm{~h}$ in $10 \%$ normal goat serum in PBT (1\% BSA, $0.5 \%$ Triton X-100 in PBS). Sections were incubated with mouse antiSV2 (DSHB, 1/10), mouse anti-synaptophysin (MAB5258, clone SY38, Millipore, 1/250) and/or mouse anti-NF (2H3, DSHB, 1/100) primary antibodies in PBT at $4^{\circ} \mathrm{C}$ overnight. Sections were washed in PBS (10 x $30 \mathrm{~min})$ and incubated with goat-anti-mouse-Alexa-488 (A28175, Invitrogen, 1/500) and a-bungarotoxin (BTX) Alexa Fluor 594 conjugate (BTXAlexa-594, B13423, ThermoFisher Scientific, 1/1000) in PBT at $4^{\circ} \mathrm{C}$ overnight. Sections were washed in PBT ( 2 x $30 \mathrm{~min})$ and PBS ( 8 x $30 \mathrm{~min})$, and mounted on microscopy slides in Vectashield mounting medium.

For quantification of the total number of endplates, BTX-labeled endplates were identified using an epifluorescence microscope (Zeiss Axio Imager Z2, 20x magnification) and a manual counter was used to count all endplates in the different focal planes of each section. Endplate numbers in all sections of the same muscle were added up to obtain the total number of endplates per muscle.

For quantification of endplate area and innervation status, sections stained with anti-SV2 (DSHB, 1/10) and a-bungarotoxin (BTX) Alexa Fluor 594 conjugate (BTX-Alexa-594, B13423, ThermoFisher Scientific, 1/1000) were imaged at a 63x magnification on a Zeiss LSM700 confocal microscope. For each muscle, two sections were imaged, and for each section, images of 5 distinct fields were obtained. Fiji software was used to obtain maximum intensity projections of a limited number of confocal sections (5 to 10), in order to generate images of a select number of individual, non-overlapping endplates. This allowed for analysis of $\sim 100$ to 150 endplates per muscle. The surface area of each of these endplates was determined using the "freehand selection" tool in Fiji to delineate endplates. To quantify innervation status, composite images of the red and green channels were generated.

Endplates that were apposed by at least three SV2-positive 'dots' were scored as innervated, 
and the percentage of innervated endplates per muscle was calculated and used for statistical analysis.

For quantification of total number of endplates in 1-month-old mouse muscle, tissue preparation was done as for newborn pups, and immunostaining was performed on freefloating $100 \mu \mathrm{m}$ longitudinal sections. Sections were permeabilized for $30 \mathrm{~min}$ in PTT, washed in PBS (3x $15 \mathrm{~min}$ ) and blocked for $1 \mathrm{~h}$ in 10\% normal goat serum, 1\% BSA, $0.5 \%$ Triton X-100 in PBS. Sections were incubated with rhodamine-conjugated BTX (Sigma, T0195, 1/1000) for $2 \mathrm{~h}$, washed in PBS (3 x $15 \mathrm{~min}$ ) and mounted on microscopy slides in Fluorsave mounting medium (Vector, H-1000).

Muscle fiber number in newborn mice-The EDL and TA muscles were used for quantification of the total number of muscle fibers, as this muscle is smaller than the TA or the gastrocnemius, with a lower number of muscle fibers, what makes the quantification of the total number of muscle fibers practically feasible. Furthermore, all fibers of the EDL muscle run from tendon to tendon, so that a cross section through the middle part of the muscle allows for quantification of the total number of muscle fibers. EDL muscles were freshly dissected and directly embedded in OCT compound (Sakura) following a "cryo-nofix' procedure. $16 \mu \mathrm{m}$ transverse sections were made using a cryostat and stored at $-80^{\circ} \mathrm{C}$ or immediately used for immunostaining using anti-dystrophin antibodies to selectively label muscle plasma membranes.

For dystrophin immunostaining, muscle sections were incubated at room temperature and hydrated with PBS ( 2 x $10 \mathrm{~min}$ ). Sections were briefly fixed (15 min) in 4\% PFA in $0.1 \mathrm{mM}$ sucrose, washed with PBS ( 2 x $10 \mathrm{~min})$, and incubated with mouse anti-dystrophin (MANDRA1, DSHB, 1/20) primary antibody in PBS with $2 \%$ donkey serum and $0.1 \%$ Triton X-100 at $4^{\circ} \mathrm{C}$ overnight. Sections were subsequently washed with PBS ( $\left.2 \times 10 \mathrm{~min}\right)$, followed by Alexa-568-conjugated anti-mouse secondary antibody (A-11031, ThermoFisher Scientific) in PBS with $2 \%$ donkey serum and $0.1 \%$ Triton $\mathrm{X}-100$ for $2 \mathrm{~h}$ at room temperature. Sections were washed in PBS (3 x $10 \mathrm{~min})$ and mounted in mounting medium with DAPI.

For quantification of the total number of muscle fibers, dystrophin-stained sections were imaged at a 10x magnification on a Zeiss LSM700 or Leica SP8 (Leica Microsystems) confocal microscope. Fiji software was used to count the total number of muscle fibers per section (multi-point selection tool).

NMJ morphology in adult mice-Mice were anesthetized with intraperitoneal injection of $100 \mathrm{mg} / \mathrm{kg}$ ketamine chlorhydrate and $5 \mathrm{mg} / \mathrm{kg}$ xylazine and transcardially perfused with 4\% PFA in 0.1 M phosphate buffer $\mathrm{pH}$ 7.4. Gastrocnemius and TA muscles were dissected, post-fixed overnight in $4 \%$ PFA at $4^{\circ} \mathrm{C}$, and washed three times with PBS. Muscles were 'teased' into muscle fiber bundles and stained overnight at room temperature with rabbit polyclonal antibodies against neurofilament (custom made, 1/100) and synaptophysin (custom made, 1/100) in PBS-0.1\% Triton X-100. After three washes with PBS-0.1\% Triton $\mathrm{X}-100$, muscles were stained with Alexa-488-conjugated donkey anti-rabbit secondary antibody (Jackson, 711-547-003, 1/500), rhodamine-conjugated BTX (Sigma, T0195; 1/500) 
and hoechst (Sigma, B2261, 1/1000). Finally, muscles were washed and mounted in aquapolymount (Polysciences, 18606-5).

Z-stack images ( $2 \mu \mathrm{m}$ optical section, objective x63) were acquired under a confocal microscope (Leica SP5, Leica Microsystems). Excitation rays were sequential argon laser $488 \mathrm{~nm}$, diode $561 \mathrm{~nm}$, Helium Neon laser $633 \mathrm{~nm}$. Emission bandwidths were $500-550 \mathrm{~nm}$ for Alexa488, 570-620nm for Alexa594, and 460nm for Hoechst. Endplate area of $100-150$ NMJ was quantified using ImageJ software and innervation status was evaluated based on the alignment of juxtaposed pre- and postsynapses. NMJs with $>80 \%$ of innervation were classified as innervated, $<80 \%$ as partially denervated and $<1 \%$ as denervated.

\section{NMJ innervation status and endplate surface area in 28-month-old mice-}

Gastrocnemius and TA muscles were dissected, post-fixed overnight in $4 \%$ PFA at $4{ }^{\circ} \mathrm{C}$, and washed three times with PBS. Muscles were 'teased' into muscle fiber bundles, incubated in blocking buffer (10\% normal goat serum, $1 \%$ BSA, and $0.5 \%$ Triton X-100 in PBS) prior to staining overnight at $4^{\circ} \mathrm{C}$ with antibodies against neurofilament $(2 \mathrm{H} 3$, DSHB, 1/100) and synaptic vesicle glycoprotein 2A (SV2, DSHB, 1/20) in blocking buffer. After three washes with PBS, muscles were stained with Alexa-405-conjugated anti-mouse secondary antibody (ThermoFisher Scientific, A31553, 1/300) and rhodamine-conjugated BTX (Sigma, T0195, 1/500). Finally, muscles were washed and mounted in Fluorsave (Vector, H-1000).

MyoD-Cre efficiency in newborn mice-To evaluate the efficiency of $M y o D^{i C r e}$ mice ${ }^{16,17}$ to excise loxP-flanked genomic sequences, $M y o D^{i C r e /+}$ mice were crossed to $R O S A^{m T / m G}$ reporter mice ${ }^{18}$. Adult $M y o D^{i C r e /+} ; R O S A^{m T / m G} /+$ (experimental) and $R O S A^{m T / m G} /+$ (control) mice were anesthetized by i.p. injection of a mixture of ketamine $(100 \mathrm{mg} / \mathrm{kg})$ and xylazine $(10 \mathrm{mg} / \mathrm{kg})$. TA muscles were dissected and fixed for $2 \mathrm{~h}$ in $4 \%$ PFA in PBS on ice. Muscles were washed in PBS (3 x $15 \mathrm{~min}$ ), cryoprotected in 30\% sucrose in PBS and embedded in OCT compound (Sakura) before cryostat sectioning $(20 \mu \mathrm{m})$.

For immunostaining, slides were incubated for $20 \mathrm{~min}$ in antigen retrieval buffer ( $2.94 \mathrm{~g}$ TriSodium citrate and $0.5 \mathrm{ml}$ Tween 20 in $1 \mathrm{~L}$ distilled water, $\mathrm{pH}$ 6) that had been heated in a microwave till boiling. Slides were washed in PBS ( 2 x $10 \mathrm{~min})$ and incubated with chicken anti-GFP (Aves Labs, GFP-1020, 1/300) and rabbit anti-RFP (MBL Life Science, PM005, $1 / 250$ ) primary antibodies in $2 \%$ donkey serum $/ 0.1 \%$ Triton $\mathrm{X}-100$ in PBS at $4{ }^{\circ} \mathrm{C}$ overnight. Next, slides were washed in PBS ( 2 x $10 \mathrm{~min}$ ) and incubated with Alexa-488-conjugated goat anti-chicken (A-11039, Invitrogen) and Alexa-568-conjugated goat anti-rabbit (A-11011, Invitrogen) secondary antibodies (1/300) in PBS with 2\% donkey serum and $0.1 \%$ Triton X-100 for $2 \mathrm{~h}$ at room temperature. Finally, slides were washed in PBS ( $2 \times 10$ min) and incubated with Alexa Fluor 647 phalloidin (ThermoFisher Scientific, A22287, $1 / 20$ ) at room temperature for $15 \mathrm{~min}$, rinsed in PBS (5 min) and mounted in DAPIcontaining mounting medium (Vectashield).

FUS/phalloidin/DAPI staining on newborn muscles-To evaluate the efficiency of $M y o D^{i C r e}$ mice to revert the Fus ${ }^{\Delta N L S}$ allele to wild type, $M y o D^{i C r e /+}$ males were crossed to $\mathrm{FuS}^{\Delta N L S /+}$ females, and timed matings were set up between $\mathrm{MyoD}^{\mathrm{iCre} /+}$; Fus ${ }^{\Delta N L S /+}$ males in the offspring and $F u s^{\Delta N L S /+}$ females. Fixed TA muscles were dissected from $M y o D^{i C r e /+}$; 
$\mathrm{FuS}{ }^{\Delta N L S / \triangle N L S}$ (experimental), Fus ${ }^{4 N L S / \triangle N L S}$ and $M y o D^{i C r e / t}$ (control) pups, washed in PBS and incubated overnight in $30 \%$ sucrose in PBS at $4^{\circ} \mathrm{C}$.

Muscles were embedded in OCT and $16 \mu \mathrm{m}$ thick cryosections were made with a cryostat. For immunostaining, sections were incubated at room temperature and hydrated with PBS (2 x 10 min). Sections were incubated with rabbit anti-FUS (Sigma, HPA008784, 1/150) primary antibody in PBS with $2 \%$ donkey serum and $0.1 \%$ Triton X-100 at $4{ }^{\circ} \mathrm{C}$ overnight. Sections were subsequently washed with PBS ( 2 x $10 \mathrm{~min}$ ), followed by Alexa-488conjugated anti-rabbit secondary antibody (A27034, Invitrogen) in PBS with 2\% donkey serum and $0.1 \%$ Triton X-100 for $2 \mathrm{~h}$ at room temperature. Sections were washed in PBS (3 x $10 \mathrm{~min}$ ) and incubated with Alexa Fluor 647-conjugated phalloidin (Cell Signaling, 8940, 1/20) for $30 \mathrm{~min}$ at room temperature. Sections were washed in PBS (3 x $10 \mathrm{~min})$ and mounted in mounting medium with DAPI (VectaShield) for confocal imaging.

FUS immunostaining in adult muscles-Unfixed muscles embedded in OCT (TissueTek® O.C.T. Compound, Sakura, 4583) were frozen in melting isopentane. Serial cuts of $14 \mu \mathrm{m}$ thick were made with cryostat (Leica CM 3050S) and deposited on Superfrost ${ }^{\circledR}$ slides (Superfrost, VWR, 631-0448). Slides were dried 20 min then fixed with 4\% PFA in $0.1 \mathrm{mM}$ sucrose $15 \mathrm{~min}$ at room temperature. After washes with PBS, AChR clusters were stained with rhodamine-conjugated BTX (Sigma, T0195; 1/500) during $1 \mathrm{~h}$ at room temperature. Unspecific binding sites were blocked with $0.1 \%$ TritonX-100 and 3\% BSA in PBS, $2 \mathrm{~h}$ at room temperature. Rabbit anti-FUS antibody (Bethyl, A300-294A, 1:1000) was incubated overnight at $4{ }^{\circ} \mathrm{C}$. After rinsing in PBS, donkey anti-rabbit Alexa Fluor 488 (Jackson, $711-547-003,1 / 500)$ was incubated $2 \mathrm{~h}$ at room temperature. Finally, sections were washed with PBS followed by water and mounted in aquapolymount (Polysciences, 18606-5).

FUS staining intensity in extrasynaptic and subsynaptic nuclei was measured using ImageJ software. Subsynaptic nuclei were defined as nuclei directly underneath alpha-BTX staining (labeling AChR) (i.e. overlapping in a confocal stack), while other nuclei were considered as extrasynaptic. The criteria for inclusion of synapses for analysis were that an endplate was clearly visible on confocal images, and that subsynaptic nuclei were present. FUS staining intensity was determined in all nuclei present in a confocal image stack. Mean FUS staining intensity in subsynaptic nuclei was then divided by FUS staining intensity in nonsynaptic nuclei, resulting in a ratio of FUS enrichment in synaptic nuclei. The resulting ratio (enrichment score) reflects the relative enrichment of FUS in subsynaptic nuclei: a ratio of 1 means no enrichment (FUS staining similar in subsynaptic and extrasynaptic nuclei), while a ratio of e.g. 3 indicates a 3-fold enrichment of FUS in subsynaptic nuclei.

Axotomy experiments-For axotomy, intraperitoneal injection of $100 \mathrm{mg} / \mathrm{kg}$ ketamine chlorhydrate and $5 \mathrm{mg} / \mathrm{kg}$ xylazine was performed on wild type C57B16/J mice. 1-2 mm of sciatic nerve was removed and lidocaine was deposited on the surgical wound. Skin was sutured and mice were daily surveyed until sacrifice 5 days after surgery.

\section{Electron microscopy}

Gastrocnemius muscles of newborn mice were fixed by immersion in $2.5 \%$ glutaraldehyde and $2.5 \%$ PFA in cacodylate buffer $(0.1 \mathrm{M}, \mathrm{pH} 7.4)$, postfixed in $1 \%$ osmium tetroxide in 
$0.1 \mathrm{M}$ cacodylate buffer for $1 \mathrm{~h}$ at $4^{\circ} \mathrm{C}$ and dehydrated through graded alcohol (50, 70, 90, 100\%) and propylene oxide for 30 min each. Samples were embedded in Epon 812. Ultrathin sections were cut at $70 \mathrm{~nm}$, contrasted with uranyl acetate and lead citrate and examined at $70 \mathrm{kv}$ with a Morgagni 268D electron microscope. Images were captured digitally by Mega View III camera (Soft Imaging System).

\section{Electromyography with repetitive nerve stimulation}

12-month-old mice were anesthetized with intraperitoneal injection of $100 \mathrm{mg} / \mathrm{kg}$ ketamine chlorhydrate and $5 \mathrm{mg} / \mathrm{kg}$ xylazine. Repetitive nerve stimulation was performed by supramaximally stimulating the sciatic nerve and detecting responses in the gastrocnemius. 3 trains of 10 stimuli were delivered at different frequency: 3, 10, 20 and $50 \mathrm{~Hz}$. The maximal amplitude (negative + positive peak) of the CMAP was measured using ImageJ software and the percentage decrement between first and tenth stimulus of each train was calculated.

\section{C2C12 myoblast culture experiments}

C2C12 myoblast cells were purchased from ATCC (ATCC® CRL-1772 ${ }^{\mathrm{TM}}$ ). Cells were cultured in Dulbecco's modified Eagle's medium containing 10\% Fetal Bovine Serum (Fisher scientific, 11531831) and 1\% Penicillin-Streptomycin (Sigma, P4333) at $37^{\circ} \mathrm{C}$ in an incubator with $5 \% \mathrm{CO}_{2}$. Between 60 and $80 \%$ of confluency, myoblasts were differentiated in myotubes with differentiation medium (identical medium, with $0.1 \%$ Fetal Bovine Serum). Culture medium was changed every day and transfections were performed between passages 5 and 15 .

siRNA Transfection-C2C12 were cultured in 6-well plates until $60 \%$ of confluency. Cells were transfected with siRNA in differentiation medium using Lipofectamine RNA iMAX (ThermoFischer Scientific, 13778150) according to the manufacturer's instructions. siRNA against FUS and negative control siRNA were provided by Dharmacon (respectively D-001810-10-20 and L-051741-00-0050). Cells were harvested 48h after transfection.

Plasmid Transfection and luciferase assay-C2C12 were cultured in 24 well-plates until $80 \%$ of confluency. Transfection was performed in differentiation medium with expression and reporter plasmids using TransIT-X2 (MIR6000, Myrus) according to the manufacturer's instructions. Expression vectors used for transfections were: pCMV empty plasmid, pCMV-Myc-FUS (expressing N-terminal myc-tagged human wild type FUS) and pCMV-Myc-FUS-R495X. Reporter plasmids were obtained from L. Schaeffer (Lyon, France $)^{5}$. After $24 \mathrm{~h}$ of transfection, proteins were extracted and luciferase activity was measured (Promega, E4550) and normalized by total proteins measured with BCA assay (Interchim, UP95424A, UP95425A).

Plasmid Transfection and immunostaining-C2C12 were cultured in 24 well-plates until $80 \%$ of confluency. Transfection was performed in differentiation medium with expression vectors using TransIT-X2 (MIR6000, Myrus) according to the manufacturer's instructions. Expression vectors used for transfections were: pCMV-Myc-FUS (expressing N-terminal myc-tagged human wild type FUS), pCMV-GFP-FUS and pCMV-Myc-FUSR495X obtained from J. Weishaupt (Ulm Germany) and N. Charlet Berguerand (Strasbourg, 
France). $24 \mathrm{~h}$ after transfection, cells were processed for immunocytochemistry. For analysis, 10 images per condition were used for quantification using ImageJ software.

24h after transfection, cells were fixed in 4\% PFA during 10min and then washed with PBS 1X. Transfected cells incubated with anti-Myc antibody (Sigma, M4439, 1/250) overnight. After three washes with PBS-0.1\% Triton X-100, cells were incubated with Alexa-594conjugated donkey anti-mouse secondary antibody (Molecular probes, A21203, 1/500) and hoechst (Sigma, B2261, 1/1000) for $2 \mathrm{~h}$ at room temperature. Finally, cells were washed with PBS followed by water and mounted in aquapolymount (Polysciences, 18606-5).

\section{RNA extraction and quantitative reverse transcription-polymerase chain}

reaction-Total RNA was extracted from $\mathrm{C} 2 \mathrm{C} 12$ or muscles (tibialis or gastrocnemius) of mice using TRIzol® reagent (Life Technologies). $1 \mu \mathrm{g}$ of RNA was reverse transcribed with iScript $^{\mathrm{TM}}$ reverse transcription (Biorad, 1708841). Quantitative polymerase chain reaction was performed using Sso Advanced Universal SYBR Green Supermix (Bio-Rad) and quantified with Bio-Rad software. Gene expression was normalized by calculating a normalization factor using H2A Histone Family Member X (H2AX), Histone Cluster 1 H2A Family Member C (H2AC), Histone Cluster 1 H2B Family Member C (H1H2BC) genes according to GeNorm software ${ }^{33}$.

Primer sequences for RT-qPCR in mouse cells and tissues

H2AX: F-TCCTGCCCAACATCCAGG, R-TCAGTACTCCTGAGAGGCCTGC

H2AC: F-CAACGACGAGGAGCTCAACAAG, R-GAAGTTTCCGCAGATTCTGTTGC

H1H2BC: F-AACAAGCGCTCGACCATCA, R-GAATTCGCTACGGAGGCTTACT

Chrna1: F-CCACAGACTCAGGGGAGAAG, R-AACGGTGGTGTGTGTTGATG

Chrnb1: F-GGCAAGTTCCTGCTTTTCGG, R-CGTCCGGAAGTGGATGTTCA

Chrng. F-GAGAGCCACCTCGAAGACAC, R-GACCAACCTCATCTCCCTGA

Chrnd: F-CGC-TGC-TTC-TGC-TTC-TAG-GG, R-ATCAGTTGGCCTTCGGCTT

Chrne: F-CAATGCCAATCCAGACACTG, R-CCCTGCTTCTCCTGACACTC

\section{Chromatin immunoprecipitation}

$1.08 \times 10^{7}$ of $\mathrm{C} 2 \mathrm{C} 12$ cells differentiated during $48 \mathrm{~h}$, were washed with PBS and fixed in $1 \%$ PFA during $10 \mathrm{~min}$. Glycine $(0.125 \mathrm{M})$ was added to stop the crosslinking reaction. After washes in PBS, cells were detached, centrifuged and resuspended in nuclei buffer $(50 \mathrm{mM}$ TrisHCl pH8, 2mM EDTA pH8, 0.1\%NP40, 10\% glycerol, 1M Na butyrate, protease inhibitor 1x). After centrifugation, nuclei were resuspended in sonication buffer $(50 \mathrm{mM}$ TrisHCl pH8, $10 \mathrm{mM}$ EDTA, 0.3\% SDS, $1 \mathrm{M}$ Na butyrate, protease inhibitor 1x) and sonicated with Diagenode Bioruptor (B0102001 - 3 Cycles of 5 min - 30s on/ 30s off). A fraction of sonicated chromatin was reverse cross linked and fragment size was checked. We used chromatin with fragment size $200-500 \mathrm{bp}$, and saved $0.3 \mu \mathrm{g}$ as $1 \%$ input. $30 \mu \mathrm{g}$ of 
chromatin was used for immunoprecipitation. Sonicated chromatin was incubated overnight at $4^{\circ} \mathrm{C}$ with $10 \mu \mathrm{g}$ of primary antibodies against FUS (FUS-1: A300-294A - Bethyl, FUS-2: A300-293A - Bethyl), or without antibody as negative control. Addition of $50 \mu \mathrm{l}$ of protein $\mathrm{A} / \mathrm{G}$ beads (53133, ThermoFisher Scientific) during $2 \mathrm{~h}$, followed by centrifugation and washes allowed chromatin immunoprecipitation. Beads were then resuspended in elution buffer (10 mM Tris pH 8, 1 mM EDTA pH 8), and chromatin was reverse cross linked (0.2 $\mathrm{M} \mathrm{NaCl}, 50 \mu \mathrm{g} / \mathrm{ml}$ of RNase, proteinase $\mathrm{K} 0.15 \%$ ) during $3 \mathrm{~h}$ at $65^{\circ} \mathrm{C}$. DNA purification was performed with QIAquick ® PCR Purification Kit (28106 - Qiagen).

Primer sequences for ChIP-PCR in C2C12 cells:

Chnra: F-GACAAGCCTCTGACTCATGATCTATGT, R-GCTGCCGGTCCTACTCCACCCTGGCT

Chnrd: F-TCCTGCCTGGGATCTTTTCGTTCTGCCCTTGG,

R-GGTTTGTCTTCCCTTCAGCCTGTTGCTGTGGA

Chnre: F-GATGACAGGCCTTGTGGATT, R-GACAAGCTTGAGGGAACAGG

Mecp2 (positive control for Fus): F- AGTATGACTCTTCCTTACCAGGAG, R- CTGATTCAAATTTGGCTCCCCT,

$H 2 A C$ (negative control for Fus): F-CGCGAAACTTGCGTTTTCAG,

\section{R- TTGGTGGTTATTTGGCCCCT.}

Data were presented normalized to $1 \%$ input.

\section{Western blotting}

Cells were washed in 1x PBS and lysed in RIPA buffer (Tris 50mM, NaCl 150mM, EDTA $1 \mathrm{mM}$, SDS $0.1 \%$, Deoxycholate $0.5 \%$, Triton X-100) containing protease inhibitor (Sigma P8340) and phosphatase inhibitor cocktail (Sigma 8345). After centrifugation at 10,000 rpm, $4^{\circ} \mathrm{C}$ during $10 \mathrm{~min}$, supernatant was saved and used to dose protein extract by using a BCA Assay (Interchim, UP95424A, UP95425A). Proteins were denatured and SDS PAGE was performed with $25 \mu \mathrm{g}$ of protein on criterion TGX stain free gel 4-20\% (Biorad, 5678094). Proteins were blotted on nitrocellulose membrane using semi-dry Transblot Turbo system (BioRad, France) and blocked with 10\% non-fat milk during $1 \mathrm{~h}$. Anti-FUS antibody (Proteintech, 11570-1-AP, 1:1000) was incubated overnight at $4^{\circ} \mathrm{C}$ in 3\% non-fat milk. Washing proceeded with washing buffer (1 M Tris pH 7.4, $\mathrm{NaCl}$ 5M, Tween $20100 \%)$ and goat anti-rabbit HRP (Abliance, BI2407, 1/5000) and was incubated $1.5 \mathrm{~h}$ at room temperature. After successive washes, proteins were visualized with chemiluminescence using ECL Lumina Forte (Millipore, France) and chemiluminescence detector (Bio-Rad, France). Total proteins were detected with stain free gel capacity (Biorad, 5678094) and used to normalized. 


\section{Experiments on human iPSC- derived motor neurons and myotubes or human skeletal muscle biopsies}

Patients from which a hair sample was obtained-FUS1 (male, 26) harbored a novel $1 \mathrm{bp}$ deletion (c.1483delC) leading to a frameshift ${ }^{34-36}$ and the modification of the protein transcript before the stop codon (R495QfsX527) within a highly conserved RGGrich region. FUS2 (male, 19) carried the frameshift mutation c.1504delG, leading to an extended translation into the 3'-UTR of the protein (D502TfS*27). Mutations in FUS1 and FUS2 displayed a juvenile ALS onset. All FUS-ALS patients showed a spinal disease onset with a bulbar progression for FUS2. ALS-related mutations within the FUS gene were localized at the C-terminus of the transcript, affecting the NLS of the protein. This information is included in Supplementary Table 1.

Reprogramming of human keratinocytes and cultivation of hiPSCs-Generation of human iPS cells from reprogrammed human keratinocytes was performed as previously described $^{37,38}$, using a lentiviral multicistronic vector ${ }^{39}$. All cell lines used were already characterized and previously published ${ }^{20,40,41}$. Briefly, plucked human hair samples were cultivated in conditioned MEF medium supplemented with $50 \mu \mathrm{g} / \mathrm{ml}$ ascorbic acid (Sigma, A4403), 10ng/ml FGF2 (Cell Guidance Systems, GFH146-50) and $10 \mu$ M Rho-associated kinase (ROCK) inhibitor (Ascent Scientific, Asc-129). After outgrow of keratinocytes, medium was changed to Epilife (Gibco, M-EPICF, S0015) supplemented with $10 \mu \mathrm{M}$ ROCK inhibitor. To obtain high reprogramming efficiency, cells were not passaged more than two times with k-dispase (BD Bioscience 354235). Viral infection was performed using polybrene (Sigma, TR-1003-G) on two consecutive days. Subsequently, cells were seeded on irradiated rat embryonic feeder cells and cultivated in hiPSC medium (Knockout DMEM, 20\% Knockout Serum Replacement (ThermoFisher Scientific, 10828028), 1\% NEAA (Gibco, 11140), 1\% GlutaMax ${ }^{\mathrm{TM}}$, 35050-038, $100 \mu \mathrm{M}$ ß-mercaptoethanol (Millipore, ES-007-E), 1\% Antibiotic Antimycotic (ThermoFisher Scientific, 15240062) supplemented with $50 \mu \mathrm{g} / \mathrm{ml}$ ascorbic acid, 10ng/ml FGF2, $10 \mu \mathrm{M}$ ROCK inhibitor). Colonies displaying iPSC morphology were mechanically lifted and cultivated under feeder free conditions with mTeSR 1 medium (Stem Cell technologies, 05850 ) at $37^{\circ} \mathrm{C}, 5 \% \mathrm{CO}_{2}$ and $5 \% \mathrm{O}_{2}$. HiPSCs were kept in culture maximally up to 35 passages.

All pluripotency tests and the spontaneous germ layer differentiation were performed as previously published or carried out according to manufacturer's protocol.

Differentiation of motor neurons-Differentiation of hiPSCs into motor neurons was performed according to published protocols $\mathrm{s}^{40,42}$. For embryoid bodies (EBs) formation, hiPSCs were cultured in suspension in EB medium (DMEM/F12 (Gibco, 31331), 20\% Knockout Serum Replacement, 1\% NEAA, 1\% $\beta$-Mercaptoethanol, $1 \%$ AntibioticAntimycotic) in ultra-low attachment flasks (Corning Costar, 734-4140). $10 \mu \mathrm{M}$ ROCK inhibitor was supplemented for the first $24 \mathrm{~h}$. Neuronal differentiation was induced by changing the medium to Differentiation medium 1 (DMEM/F12, $1 \%$ NEAA, $2 \mu \mathrm{g} / \mathrm{ml}$ heparin (Sigma, H3149), 1\% Antibiotic Antimycotic, 2\% Hormone mix (24 nM Sodium Selenite (Sigma, S9133-1MG) + $16 \mathrm{nM}$ Progesterone (Sigma, P6149-1MG) + $0.08 \mathrm{mg} / \mathrm{ml}$ Apotransferrin (Sigma, T2036-1G) $+0.02 \mathrm{mg} / \mathrm{ml}$ Insulin (SAFC, 91077C-1G) $+7.72 \mu \mathrm{g} / \mathrm{ml}$ 
Putrescin (Sigma, P7505-25MG)) supplemented with $10 \mathrm{ng} / \mathrm{ml}$ GDNF (Peprotech, 450-10), $10 \mathrm{ng} / \mathrm{ml}$ BDNF (Peprotech, 540-02), 10 ng/ml IGF-1 (Peprotech, 100-11), $0.1 \mu$ M cAMP (Sigma, D0260), $20 \mu \mathrm{g} / \mathrm{ml}$ ascorbic acid) on day 4. EBs were seeded in Differentiation medium 1 on laminin-coated (Sigma, 11243217001) plates on day 7. On day 10, medium was replaced with Differentiation medium 2 (Differentiation medium 1, containing $0.1 \mu \mathrm{M}$ of retinoid acid (Sigma, R2625-100MG)). On day 15, neurospheres were transferred into T75 low-attachment flasks containing Differentiation medium 3 (Differentiation medium1, containing 1 pumorphamine (Calbiochem, 540220), $0.1 \mu \mathrm{M}$ retinoid acid and 2\% B27 (Gibco, 12587)). At day 28, up to 3 neurospheres were seeded on 35mm $\mu$-dishes (Ibidi, 86694) pre-coated with Poly-L-Ornithin (Sigma, P4957) and coated with laminin. Medium was changed to Differentiation medium 4 (Differentiation medium 1, containing $0.05 \mu \mathrm{M}$ retinoid acid, $0.5 \mu \mathrm{M}$ pumorphamine and $2 \% \mathrm{~B} 27$ ).

Differentiation of myotubes-Differentiation of myogenic cells from human iPSCs was performed basically as previously published using a spheres-based culture ${ }^{21}$. Briefly, hiPSCs were cultured in suspension using ultra low attachment flasks in Stemline medium (Sigma, SA3194) containing $100 \mathrm{ng} / \mathrm{ml}$ FGF-2, $100 \mathrm{ng} / \mathrm{ml}$ EGF and $5 \mathrm{ng} / \mathrm{ml}$ heparan sulfate (Sigma, H7640-1mg). Cells were split weekly using a 2 min accutase (Sigma, A6964) digestion and media were exchanged every second day. After 6 weeks of cultivation, cells were transferred into single cells using accutase digestion and filtration through a $30 \mu \mathrm{m}$ pre-separation filter (Miltenyi Biotec, 130-041-407). 200,000 Cells per well were seeded on glass coverslips in 24 well plates, coated with PLO and laminin. Medium was changed to DMEM (Gibco, 41965) containing 2\% B27 and 1\% Antibiotic-Antimycotic with a media exchange twice per week. After 8 weeks of final differentiation mature myotubes were obtained and fixed or lysed for analysis.

To perform co-cultivation of iPSC-derived muscle cells with iPSC-derived motor neurons, myogenic cells were differentiated using Pax7-induced stem cell-derived progenitors, as described $^{22,43}$.

Generation of isogenic control cell line-An isogenic control cell line (CNTL3) was generated by inserting a cytosine in position 1483 and consequently correcting the mutation, using Clustered Regularly Interspaced Short Palindromic Repeats (CRISPR) technology. This cell line was previously characterized and published ${ }^{20}$.

The C-nucleotide was inserted using a customized guide RNA (TATGATCGAGGCGGCTACCGGG) and a sequence specific donor vector purchased from Genewiz, containing the respective template with the C-nucleotide insertion. DNA transfection into patient-derived iPSCs was performed using Amaxa nucleofection (Lonza, VPH-5012). Subsequently, single cell-derived colonies were screened by a PCR-based approach.

Quantitative Real-time PCR-Total RNA was isolated using the RNeasy Mini Kit (Qiagen, 74106) according to the manufacturers' protocol and eluted in $40 \mu \mathrm{l}$ RNAse-free water. 
First strand synthesis and quantitative real-time-PCR amplification were performed in a onestep, single-tube reaction using the QuantiFast SYBR Green RT-PCR kit (Qiagen, 204174) and the Rotor-Gene-Q real-time PCR machine model 2-Plex HRM (Qiagen) in a total reaction volume of $20 \mu \mathrm{l}$. Reactions were performed in $0.1 \mathrm{ml}$ strip tubes and were composed of $1 \mu \mathrm{l}$ of RNA, $10 \mu \mathrm{l}$ QuantiFast SYBR Green RT-PCR Master Mix, $0.2 \mu \mathrm{l}$ RT Enzyme, $6.8 \mu \mathrm{l}$ RNAse-free water and $2 \mu \mathrm{l}$ of QuantiTect primer $(0.5 \mu \mathrm{mol})$.

qRT-PCR cycling conditions were as follows: 1 cycle Reverse Transcription of $10 \mathrm{~min}$ at $50^{\circ} \mathrm{C}, 1$ cycle Denaturation of $5 \mathrm{~min}$ at $95^{\circ} \mathrm{C}, 40$ cycles of $5 \mathrm{sec}$ at $95^{\circ} \mathrm{C}$ for denaturation, 10 sec at $60^{\circ} \mathrm{C}$ for annealing and elongation. The cycle threshold $\left(\mathrm{C}_{\mathrm{T}}\right)$ for fluorescence detection were calculated and set by the Rotor-Gene Q Software version 2.0.2 (Qiagen) at $10^{-2}$ norm. fluorescence. All results are shown relative to the reference-housekeeping gene hydroxymethylbilane synthase (HMBS) to obtain values for relative quantification. QuantiTect Primer pairs were purchased from Qiagen.

Immunocytochemistry-For immunocytochemistry, cultures were fixed using $4 \%$ paraformaldehyde (Merck) and 10\% sucrose (Roth, 4621.1) in phosphate buffered saline (DPBS, Gibco, H15-001) for $15 \mathrm{~min}$. After washing, samples were permeabilized with $0.2 \%$ Triton X-100 (Roche, 10789704001) for $10 \mathrm{~min}$. After blocking with 5\% FBS (Gibco, 10500 ) and $10 \%$ goat serum (Millipore, S26-100mL) in DPBS for $1 \mathrm{~h}$, primary antibodies rabbit anti-FUS (Bethyl Labs, Montgomery, A300-294A, 1;1000), rabbit anti-FUS (Sigma, HPA008784, 1:1000) chicken anti-NF-H (Antibodies online, ABIN1842223, 1:10.000), mouse anti-actinin (Sigma, A7811, 1:500), mouse anti-Desmin (Dako, M0760, 1:500), rabbit anti-Myosin heavy chain (R\&D Systems, MAB4470, 1:500), mouse anti-MyoG (Millipore, MAB3876, 1:500), mouse anti-Dihydropyridine Receptor alpha (Millipore, MAB427, 1:500), rabbit anti-Ryanodine receptor (Millipore, AB9078, 1:500), rhodamineconjugated BTX (Sigma, T0195, 1:1000) were incubated for $48 \mathrm{~h}$ at $4^{\circ} \mathrm{C}$. After subsequent washing, secondary antibodies were incubated for $1 \mathrm{~h}$ at room temperature: Alexa Fluor 488 goat anti-rabbit (A-11034), Alexa Fluor 647 goat anti-chicken (A-21449), Alexa Fluor 568 goat anti-mouse (A-110049) (all 1:500, Invitrogen). Samples were mounted with ProLong Gold Antifade reagent with DAPI (Invitrogen, P36935).

Image acquisition and analysis-Fluorescence images were obtained using an Axioscope microscope (Zeiss) equipped with a CCD camera (16bits, 1280 x 1024pixels) and the Axiovision software (Zeiss). Quantification analysis and analysis of BT positive structures were performed using ImageJ Fiji software (www.imageJ.nih.gov) ${ }^{44}$. To investigate the structures forming an individual endplate, the ImageJ Fiji plugin "Analyze Particles" was used.

Human skeletal muscle biopsies-Muscle specimens were extracted in open biopsy of vastus lateralis (FUS5), deltoid (FUS4), triceps (FUS1) and biceps (CNTRL4,5,6). Control patients presented at our Center with chronic pain syndrome, which was the reason for muscular biopsy, showing no myopathic changes in the biopsy results. FUS1 and FUS 4 had juvenile onset of the disease and clinically the disease onset for FUS1 and FUS5 was spinal and for FUS4 bulbar. 
Samples were frozen with chilled isopentane and stored at $-80^{\circ} \mathrm{C}$. Serial cuts of $10 \mu \mathrm{m}$ thick were made with cryostat (Leica CM 3050S) and deposited on Superfrost slides (Superfrost, VWR, 631-0448). Slides were dried 20 min then fixed with 4\% PFA in $0.1 \mathrm{mM}$ sucrose 15 min at room temperature. Sections were stained with Hematoxylin and Eosin or used for immunohistochemistry. After washing with PBS, AChR clusters were stained with rhodamine-conjugated BTX (Sigma, T0195; 1:500) during 1h at room temperature. Sections were then permeabilized with $0.1 \%$ Triton X-100 and nonspecific binding sites were blocked with $10 \%$ goat serum and 5\% FBS in PBS, $2 \mathrm{~h}$ at room temperature. Rabbit anti-FUS (Sigma, HPA008784, 1:1000), guinea pig anti-Synaptophysin (synaptic systems, 101004, 1:500) were incubated overnight at $4^{\circ} \mathrm{C}$. After rinsing in PBS and goat anti-rabbit Alexa Fluor 488, (Invitrogen, A-11034, 1/500) and goat anti-guinea pig Alexa Fluor 6472 (Invitrogen, A-21450) were incubated $2 \mathrm{~h}$ at room temperature. Finally, sections were washed with PBS followed by water and mounted with ProLong Gold Antifade reagent with DAPI (Invitrogen, P36935).

Confocal images were acquired with a laser scanning microscope (Leica DMi8) equipped with an ACS APO 20x, 40x or $63 x$ oil DIC immersion objective. Images were acquired with a resolution of $1024 \times 1024$ pixels.

Electromyography-Needle electromyography (EMG) was performed in all FUS-ALS patients and controls using concentric needle electrodes ( $38 \times 0.45 \mathrm{~mm}$ ). EMG was performed on a 4 channel SIGMA EMG machine by Neurowerk (Neurowerk Medizintechnik, Gelenau, Germany). In each patient and muscle, manual single potential analysis with 20 single potentials minimum per muscle was performed, it was checked whether abnormal spontaneous activity was present on at least 20 sites of the muscle and the maximal innervation pattern was recorded. The needle EMG was performed within the clinical diagnostic work-up at the University Hospital of Ulm, Germany, by an experienced neurologist.

Myogenic potentials were classified as small and/or of shorter duration according to the published lower limits for each individual muscle ${ }^{45}$ and of pathological configuration, i.e. increased number of phases or turns. Chronic neurogenic MUAP were classified as enlarged and/or of longer duration and of pathological configuration. The number of phases of a MUAP was considered pathological if $>4$ and the number of turns if $>54,46$. Changes were only classified as pathological (i.e. myogenic or chronic neurogenic) if $>15 \%$ of the MUAP analyzed fulfilled these criteria.

\section{Statistics}

All results from analysis are presented as mean \pm standard error of the mean (SEM) and differences were considered significant when $\mathrm{p}<0.05$. No statistical methods were used to pre-determine sample sizes, but our sample sizes are similar to those reported in previous publications ${ }^{14,15}$. Whenever possible, data collection and analysis were performed by investigators blinded to the genotype of the mice, cells or patients. Animals or samples were assigned to the various experimental groups based on their genotype. For mouse experiments, littermate controls were used whenever possible. For a given experiment, 
samples from the different experimental groups were processed in parallel and analyzed in random order.

Before analysis, a Robust regression and Outlier removal method (ROUT) was performed to detect all outliers. This nonlinear regression method fits a curve that is not influenced by outliers. Residuals of this fit were then analyzed by a test adapted from the False Discovery Rate approach, to identify any outliers. All data points that were considered outliers were excluded from further data analysis. Normality and homoscedasticity of all data sets was assessed by a Shapiro-Wilk and Brown-Forsythe (F-test for t-tests) test respectively. Subsequent statistical tests were only performed if all assumptions were met.

For comparison of normally distributed data of two groups, two-tailed unpaired Student's $t$ test was used in combination with F-test to confirm that the variances between groups were not significantly different. Non-parametric Mann-Whitney was performed for data not distributed normally. An unpaired t-test with Welch's correction was performed for data with unequal variances.

Comparisons of data consisting of more than two groups, varying in a single factor, were performed using (nested) one-way ANOVA or Kruskall-Wallis. Analysis of normally distributed data consisting of only individual data points (biological replicates) was performed using one-way ANOVA followed by Tukey's, Dunnett's or Bonferroni's multiple comparisons post hoc test. Normally distributed data consisting of both biological and technical replicates were analyzed using nested one-way ANOVA and subsequent Tukey's multiple comparisons test. For not normally distributed data, a non-parametric KruskallWallis followed by Dunn's multiple comparisons test was used.

Comparisons of data consisting of more than two groups, varying in two factors, was performed using two-way ANOVA and subsequent Tukey's or Bonferroni's multiple comparisons test.

For human cell culture experiments, Fisher's Exact Test followed by Bonferroni correction for multiple comparisons was used. GFP expression in muscle cells of $R O S A^{m T / m G} /+$ and $M y o D^{i C r e /+} ; R O S A^{m T / m G} /+$ mice was analyzed by a One-sample Wilcoxon signed rank test, since GFP expression in $R O S A^{m T / m G} /+$ mice was zero. For the same reason, nested Onesample Wilcoxon signed rank was also performed in FUS subcellular localization experiments after transfection with WT and mutant FUS.

Data were analyzed by using GraphPad Prism version 8.0 and R (3.5.1). Detailed statistical information for each figure, including statistical tests used and exact p-values, is shown in Supplementary Table 4.

\section{Supplementary Material}

Refer to Web version on PubMed Central for supplementary material. 


\section{Acknowledgements}

We thank Drs. L. Schaeffer (University of Lyon, France), A. Verger (Université Lille Nord de France, France), J. Weishaupt (Ulm University, Germany) and R. Perlingeiro (University of Minnesota, USA) for the kind gift of plasmids, and Dr. N. Charlet Berguerand for advice in siRNA treatment. We would like to thank the General Instruments Facility of the Faculty of Science of Radboud University, and I. Alexopoulos in particular for advice on image acquisition and analysis. The authors would like to thank S. Seltenheim, L. Dietz and R. Zienecker for excellent technical support. A. Knehr, Department of Neurology, Ulm University, for collecting human material. B. Mayer, Institute for Epidemiology and Medical Biometrics, Ulm University, for advice on statistical analysis. We are grateful to all the participants for willingly providing us with hair and muscle samples. This work was directly supported by ALS Association Investigator Initiated Awards (grants 2235, 3209 and 8075; to LD and CLT); the Frick Foundation (award 2013 to LD and CLT); Association Française contre les Myopathies (grant \#18280; to LD, CLT and ES); Virtual Helmholtz Institute "RNA dysmetabolism in ALS and FTD" (WP2, to LD, ACL and TMB), the DZNE (Ulm site) and agence nationale de la recherche (ToFU, EpiFUS, to LD), the Bundesministerium für Bildung und Forschung (BMBF01EK1611C to TMB and MD), the Max Planck Society (to ES) and the Donders Center for Neuroscience (to ES). Work in our laboratories is supported by ARSla (call 2014 and 2016 to LD), the fondation "recherche sur le cerveau" (call 2015, to LD), Axa Banque Patrimoniale (Bourse recherche maladies rares, to LD), Fondation pour la recherche médicale (Equipe FRM, to LD), the Muscular Dystrophy Association (MDA479773, to ES), the EU Joint Programme - Neurodegenerative Disease Research (JPND; grant numbers ZonMW 733051075 (TransNeuro) and ZonMW 733051073 (LocalNMD) to ES) and an ERC consolidator grant (ERC-2017-COG 770244 to ES).

\section{Data availability and biological materials}

The data that support the findings of this study and biological materials used are available from the corresponding authors upon request.

\section{References}

1. Darabid H, Perez-Gonzalez AP, Robitaille R. Neuromuscular synaptogenesis: coordinating partners with multiple functions. Nat Rev Neurosci. 2014; 15:703-718. [PubMed: 25493308]

2. Tintignac LA, Brenner HR, Ruegg MA. Mechanisms Regulating Neuromuscular Junction Development and Function and Causes of Muscle Wasting. Physiol Rev. 2015; 95:809-852. [PubMed: 26109340]

3. Shi L, Fu AK, Ip NY. Molecular mechanisms underlying maturation and maintenance of the vertebrate neuromuscular junction. Trends Neurosci. 2012; 35:441-453. [PubMed: 22633140]

4. Hippenmeyer S, Huber RM, Ladle DR, Murphy K, Arber S. ETS transcription factor Erm controls subsynaptic gene expression in skeletal muscles. Neuron. 2007; 55:726-740. [PubMed: 17785180]

5. Ravel-Chapuis A, Vandromme M, Thomas JL, Schaeffer L. Postsynaptic chromatin is under neural control at the neuromuscular junction. EMBO J. 2007; 26:1117-1128. [PubMed: 17304221]

6. Taylor JP, Brown RH Jr, Cleveland DW. Decoding ALS: from genes to mechanism. Nature. 2016; 539:197-206. [PubMed: 27830784]

7. Pun S, Santos AF, Saxena S, Xu L, Caroni P. Selective vulnerability and pruning of phasic motoneuron axons in motoneuron disease alleviated by CNTF. Nat Neurosci. 2006; 9:408-419. [PubMed: 16474388]

8. Fischer LR, et al. Amyotrophic lateral sclerosis is a distal axonopathy: evidence in mice and man. Exp Neurol. 2004; 185:232-240. [PubMed: 14736504]

9. Dadon-Nachum M, Melamed E, Offen D. The "dying-back" phenomenon of motor neurons in ALS. J Mol Neurosci. 2011; 43:470-477. [PubMed: 21057983]

10. Schwartz JC, Cech TR, Parker RR. Biochemical Properties and Biological Functions of FET Proteins. Annu Rev Biochem. 2015; 84:355-379. [PubMed: 25494299]

11. Ling SC, Polymenidou M, Cleveland DW. Converging mechanisms in ALS and FTD: disrupted RNA and protein homeostasis. Neuron. 2013; 79:416-438. [PubMed: 23931993]

12. Deng H, Gao K, Jankovic J. The role of FUS gene variants in neurodegenerative diseases. Nature Reviews Neurology. 2014; 10:337-348. [PubMed: 24840975] 
13. Dormann D, et al. ALS-associated fused in sarcoma (FUS) mutations disrupt Transportin-mediated nuclear import. EMBO J. 2010; 29:2841-2857. [PubMed: 20606625]

14. Scekic-Zahirovic J, et al. Toxic gain of function from mutant FUS protein is crucial to trigger cell autonomous motor neuron loss. EMBO J. 2016; 35:1077-1097. [PubMed: 26951610]

15. Scekic-Zahirovic J, et al. Motor neuron intrinsic and extrinsic mechanisms contribute to the pathogenesis of FUS-associated amyotrophic lateral sclerosis. Acta Neuropathol. 2017; 133:887906. [PubMed: 28243725]

16. Kanisicak O, Mendez JJ, Yamamoto S, Yamamoto M, Goldhamer DJ. Progenitors of skeletal muscle satellite cells express the muscle determination gene, MyoD. Dev Biol. 2009; 332:131141. [PubMed: 19464281]

17. Yamamoto M, et al. A multifunctional reporter mouse line for Cre- and FLP-dependent lineage analysis. Genesis. 2009; 47:107-114. [PubMed: 19165827]

18. Muzumdar MD, Tasic B, Miyamichi K, Li L, Luo L. A global double-fluorescent Cre reporter mouse. Genesis. 2007; 45:593-605. [PubMed: 17868096]

19. Tan AY, Riley TR, Coady T, Bussemaker HJ, Manley JL. TLS/FUS (translocated in liposarcoma/ fused in sarcoma) regulates target gene transcription via single-stranded DNA response elements. Proc Natl Acad Sci U S A. 2012; 109:6030-6035. [PubMed: 22460799]

20. Higelin J, et al. FUS Mislocalization and Vulnerability to DNA Damage in ALS Patients Derived hiPSCs and Aging Motoneurons. Front Cell Neurosci. 2016; 10:290. [PubMed: 28082870]

21. Hosoyama T, McGivern JV, Van Dyke JM, Ebert AD, Suzuki M. Derivation of myogenic progenitors directly from human pluripotent stem cells using a sphere-based culture. Stem Cells Transl Med. 2014; 3:564-574. [PubMed: 24657962]

22. Demestre M, et al. Formation and characterisation of neuromuscular junctions between hiPSC derived motoneurons and myotubes. Stem Cell Res. 2015; 15:328-336. [PubMed: 26255853]

23. de Carvalho M, et al. Electrodiagnostic criteria for diagnosis of ALS. Clin Neurophysiol. 2008; 119:497-503. [PubMed: 18164242]

24. So E, et al. Mitochondrial abnormalities and disruption of the neuromuscular junction precede the clinical phenotype and motor neuron loss in hFUSWT transgenic mice. Hum Mol Genet. 2018; 27:463-474. [PubMed: 29194538]

25. Sharma A, et al. ALS-associated mutant FUS induces selective motor neuron degeneration through toxic gain of function. Nature communications. 2016; 7

26. Naumann M, et al. Impaired DNA damage response signaling by FUS-NLS mutations leads to neurodegeneration and FUS aggregate formation. Nature communications. 2018; 9:335.

27. Miller TM, et al. Gene transfer demonstrates that muscle is not a primary target for non-cellautonomous toxicity in familial amyotrophic lateral sclerosis. Proc Natl Acad Sci U S A. 2006; 103:19546-19551. [PubMed: 17164329]

28. Towne C, Raoul C, Schneider BL, Aebischer P. Systemic AAV6 delivery mediating RNA interference against SOD1: neuromuscular transduction does not alter disease progression in fALS mice. Mol Ther. 2008; 16:1018-1025. [PubMed: 18414477]

29. Dobrowolny G, et al. Skeletal muscle is a primary target of SOD1G93A-mediated toxicity. Cell Metab. 2008; 8:425-436. [PubMed: 19046573]

30. Wong M, Martin LJ. Skeletal muscle-restricted expression of human SOD1 causes motor neuron degeneration in transgenic mice. Hum Mol Genet. 2010; 19:2284-2302. [PubMed: 20223753]

31. Williams AH, et al. MicroRNA-206 delays ALS progression and promotes regeneration of neuromuscular synapses in mice. Science. 2009; 326:1549-1554. [PubMed: 20007902]

32. Kedage V, et al. An Interaction with Ewing's Sarcoma Breakpoint Protein EWS Defines a Specific Oncogenic Mechanism of ETS Factors Rearranged in Prostate Cancer. Cell Rep. 2016; 17:12891301. [PubMed: 27783944]

33. Vandesompele J, et al. Accurate normalization of real-time quantitative RT-PCR data by geometric averaging of multiple internal control genes. Genome Biol. 2002; 3

34. Belzil VV, et al. Novel FUS deletion in a patient with juvenile amyotrophic lateral sclerosis. Arch Neurol. 2012; 69:653-656. [PubMed: 22248478] 
35. Japtok J, et al. Stepwise acquirement of hallmark neuropathology in FUS-ALS iPSC models depends on mutation type and neuronal aging. Neurobiol Dis. 2015; 82:420-429. [PubMed: 26253605]

36. Lenzi J, et al. ALS mutant FUS proteins are recruited into stress granules in induced pluripotent stem cell-derived motoneurons. Dis Model Mech. 2015; 8:755-766. [PubMed: 26035390]

37. Aasen T, et al. Efficient and rapid generation of induced pluripotent stem cells from human keratinocytes. Nat Biotechnol. 2008; 26:1276-1284. [PubMed: 18931654]

38. Takahashi K, Yamanaka S. Induction of pluripotent stem cells from mouse embryonic and adult fibroblast cultures by defined factors. Cell. 2006; 126:663-676. [PubMed: 16904174]

39. Warlich E, et al. Lentiviral vector design and imaging approaches to visualize the early stages of cellular reprogramming. Mol Ther. 2011; 19:782-789. [PubMed: 21285961]

40. Stockmann M, et al. Developmental and functional nature of human iPSC derived motoneurons. Stem Cell Rev. 2013; 9:475-492.

41. Linta L, et al. Rat embryonic fibroblasts improve reprogramming of human keratinocytes into induced pluripotent stem cells. Stem Cells Dev. 2012; 21:965-976. [PubMed: 21699413]

42. Hu BY, Zhang SC. Differentiation of spinal motor neurons from pluripotent human stem cells. Nat Protoc. 2009; 4:1295-1304. [PubMed: 19696748]

43. Darabi R, et al. Human ES- and iPS-derived myogenic progenitors restore DYSTROPHIN and improve contractility upon transplantation in dystrophic mice. Cell Stem Cell. 2012; 10:610-619. [PubMed: 22560081]

44. Schindelin J, et al. Fiji: an open-source platform for biological-image analysis. Nat Methods. 2012; 9:676-682. [PubMed: 22743772]

45. Bischoff C, Stalberg E, Falck B, Eeg-Olofsson KE. Reference values of motor unit action potentials obtained with multi-MUAP analysis. Muscle Nerve. 1994; 17:842-851. [PubMed: 8041391]

46. Gilai, AN. Analysis of Turns and Amplitude in EMGComputer-aided Electromyography and Expert Systems, Clinical Neurophysiology Updates. Desmedt, JE, editor. Karger; Amsterdam: 1989. 

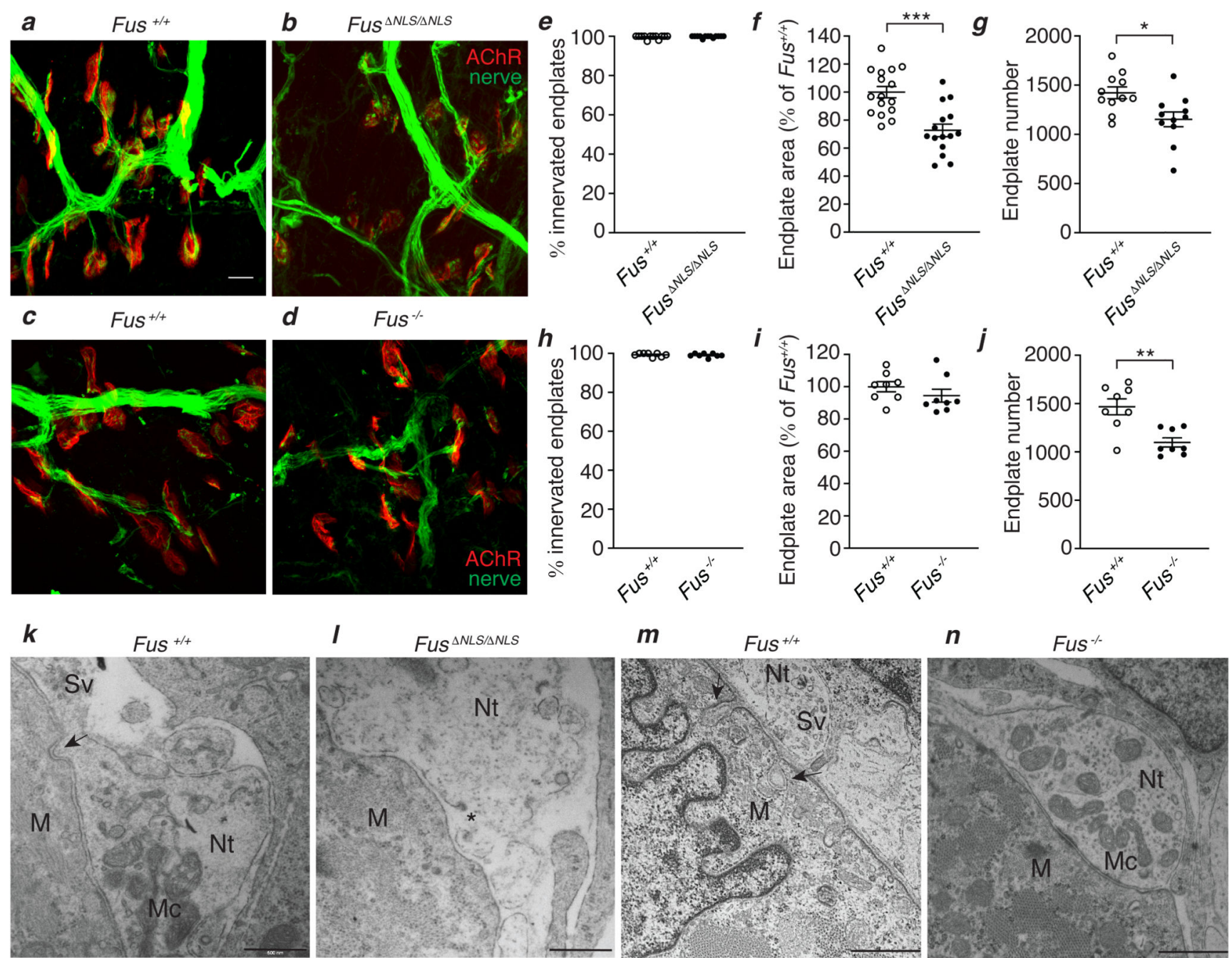

Figure 1. Neuromuscular junction (NMJ) defects in newborn Fus mutant mice.

a-d, Representative images showing NMJs in TA muscles of newborn Fus ${ }^{+/+}$(a) versus $F u s^{\Delta N L S / \triangle N L S}$ (b) and Fus ${ }^{+/+}$(c) versus Fus ${ }^{-/}$mice (d). Axons and presynaptic terminals were visualized by immunostaining with antibodies against neurofilament and SV2 (green). AChRs in muscle endplates were visualized using fluorescently labeled BTX (red). Scale bar: $10 \mu \mathrm{m}$. e-j, Dot plots showing the percentage $(\%)$ of innervated endplates $(\mathrm{e}, \mathrm{h})$, the average endplate area (as \% of $\mathrm{Fus}^{+/+}$) $(\mathrm{f}, \mathrm{i})$ and the total number of endplates $(\mathrm{g}, \mathrm{j})$ in the TA muscle of newborn Fus ${ }^{+/+}$(open circles) versus Fus $\Delta N L S / \Delta N L S$ (closed circles) (e-g) and Fus ${ }^{+/+}$(open circles) versus Fus ${ }^{-/-}$(closed circles) mice (h-j). Two-sided Mann-Whitney (e,h) or unpaired t-test (f,g,i,j); ${ }^{*} \mathrm{p}=0.011, * * \mathrm{p}=0.0017, * * * \mathrm{p}<0.0001 ; \mathrm{N}=12 \mathrm{Fus}^{+/+}$versus 13 $F u s^{\Delta N L S / \Delta N L S}(\mathrm{e}), 16 \mathrm{Fus}^{+/+}$versus $15 \mathrm{Fus}^{\Delta N L S / \Delta N L S}$ (f), $11 \mathrm{Fus}^{+/+}$versus $11 \mathrm{Fus} \mathrm{s}^{\Delta N L S / \Delta N L S}$ (g), $8 \mathrm{Fus}^{+/+}$versus $8 \mathrm{Fus}^{-/-}(\mathrm{h}, \mathrm{i}, \mathrm{j})$ mice. Average \pm SEM. k-n, Representative TEM images of NMJs in E18.5 gastrocnemius muscle of Fus ${ }^{+/+}(\mathrm{k})$ versus Fus $s^{\Delta N L S / \Delta N L S}(\mathrm{l})$ and Fus $s^{+/+}$ (m) versus $\mathrm{Fus}^{-/-}$(n) mice. Arrows indicate junctional folds; * indicates membrane disruption. Nt: nerve terminal; M: muscle; Mc: mitochondria; Sv: synaptic vesicles. 5 Fus 
${ }^{+/}, 4 \mathrm{FuS}^{-/-}$and $5 \mathrm{Fu} S^{\Delta N L S / \triangle N L S}$ mice were analyzed in a single experiment. Scale bar: $0.5 \mu \mathrm{m}(\mathrm{k}, \mathrm{l})$ and $1 \mu \mathrm{m}(\mathrm{m}, \mathrm{n})$. 


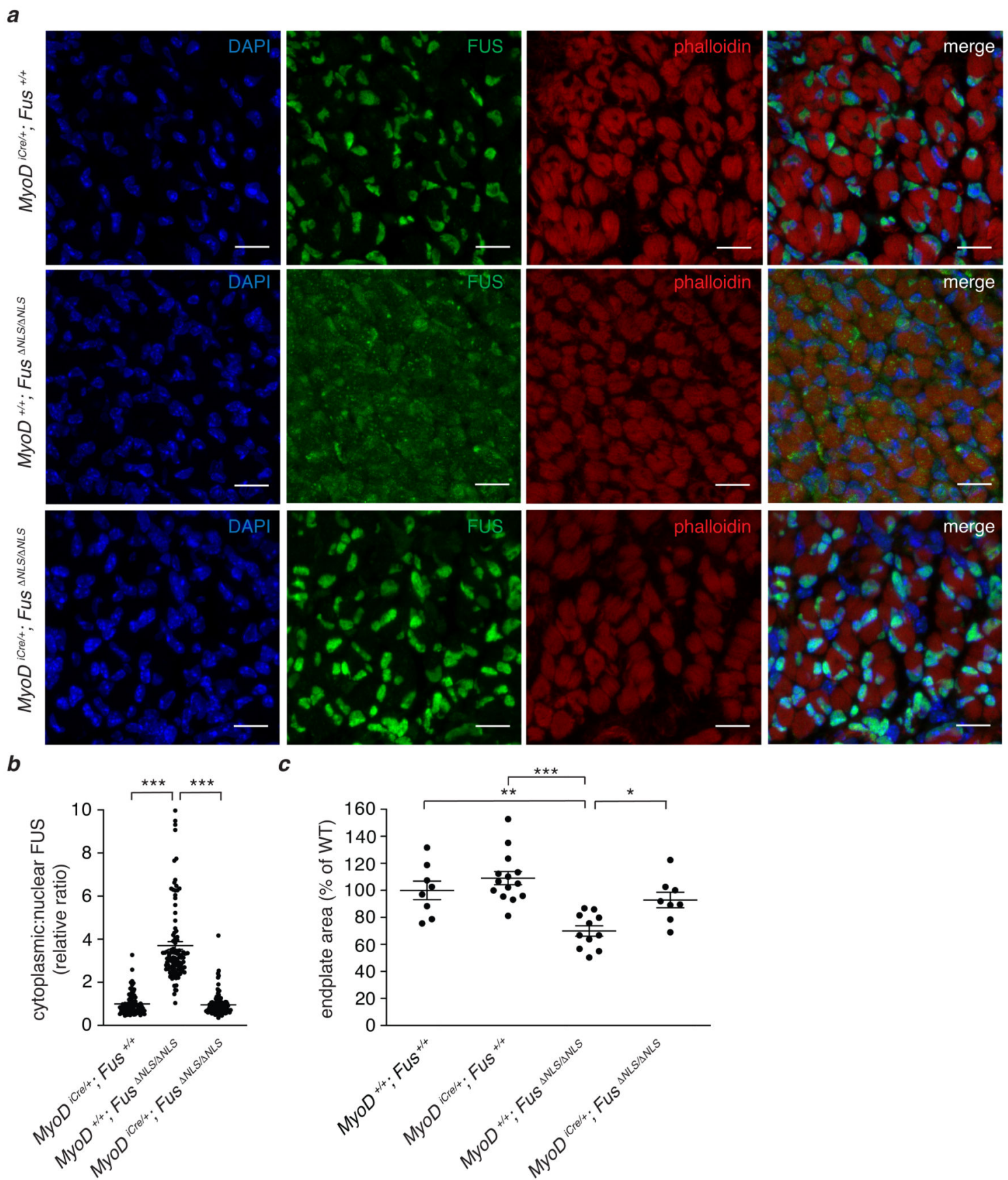

Figure 2. Cytoplasmic mislocalized FUS ${ }^{\Delta \mathrm{NLS}}$ protein is intrinsically toxic to muscle.

a, immunostaining of FUS in the TA of newborn $\mathrm{MyoD}^{i C r e /+} ; \mathrm{Fus}^{+/+}$(upper row), $\mathrm{MyoD}^{+/+}$; Fus ${ }^{\triangle N L S / \triangle N L S}$ (middle row), and $M y o D^{i C r e /+} ; F u s^{\Delta N L S / \triangle N L S}$ (lower row) mice. Nuclei were labeled with DAPI and myofibers with the filamentous actin probe phalloidin. Merged images are shown in the right column. Scale bar: $15 \mu \mathrm{m}$. b, Ratio of cytoplasmic:nuclear FUS distribution, relative to $\mathrm{MyOD}^{i \mathrm{Cre} /+} ; \mathrm{Fus}^{+/+}$(= control set at 1). Kruskal-Wallis with Dunn's multiple comparisons test; ***p<0.0001; N=81 MyoD $^{i C r e /+} ;$ Fus $^{+/+}, 101 \mathrm{MyoD}^{+/+}$; Fus ${ }^{\Delta \mathrm{NLS} / \Delta \mathrm{NLS}}, 103 \mathrm{MyoD}^{i C r e /+} ; \mathrm{Fus}{ }^{\Delta N L S / \Delta N L S}$ muscle cells from 3 TA sections per 
genotype. Average \pm SEM. c, Endplate surface area as $\%$ of wild type littermate controls in TA of newborn offspring from $\mathrm{MyOD}^{i \mathrm{Cre} /+} ; \mathrm{FuS}^{4 N L S /+} \times \mathrm{FuS}^{4 N L S /+}$ crosses. Two-way ANOVA with Tukey's multiple comparisons test; $* \mathrm{p}=0.027, * * \mathrm{p}=0.0024, * * * \mathrm{p}<0.0001$; $\mathrm{N}=8 \mathrm{MyoD}^{+/+} ; \mathrm{Fus}^{+/+}, 14 \mathrm{MyoD}^{\text {iCre/+}} ; \mathrm{FuS}^{+/+}, 11 \mathrm{MyoD}^{+/+} ; \mathrm{Fus}^{\Delta \mathrm{NLS} / \Delta \mathrm{NLS}}, 8 \mathrm{MyoD}^{\mathrm{iCre} /+}$; $F u S^{\Delta N L S / \triangle N L S}$ mice. Average \pm SEM. 


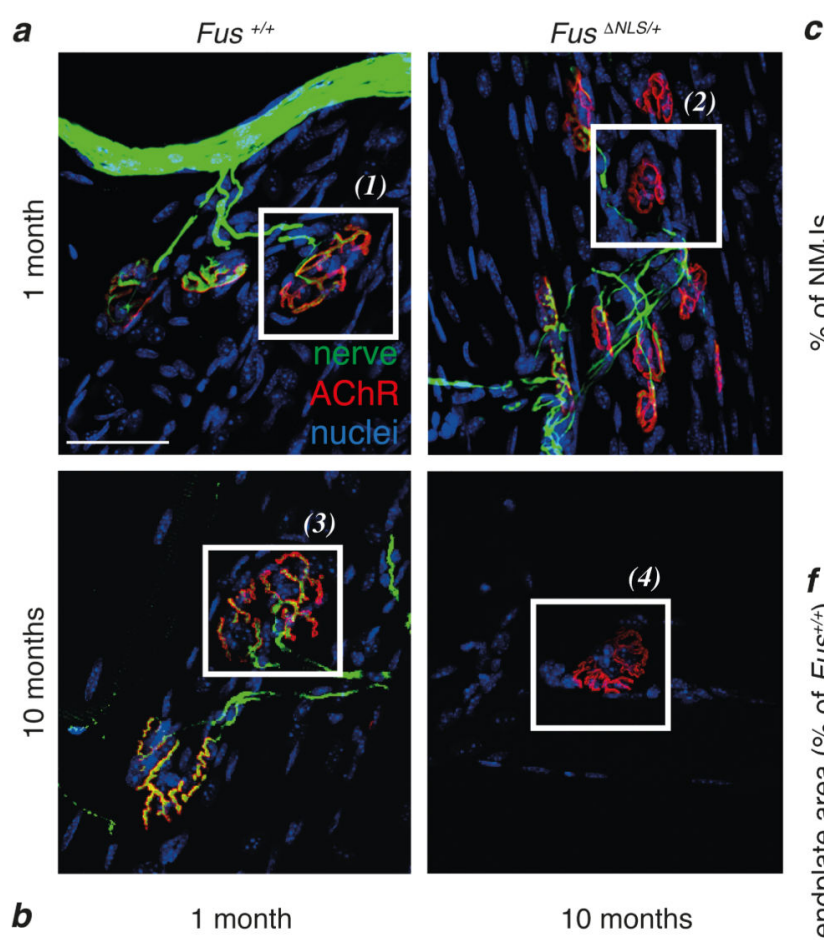

b
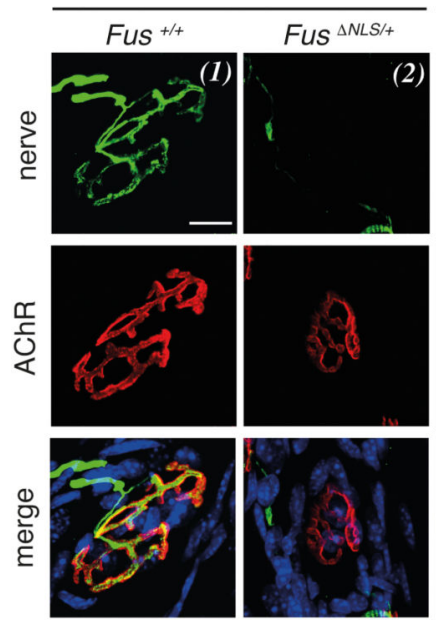
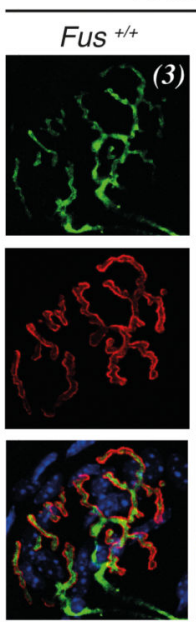

$\boldsymbol{c} \square$ innervated d

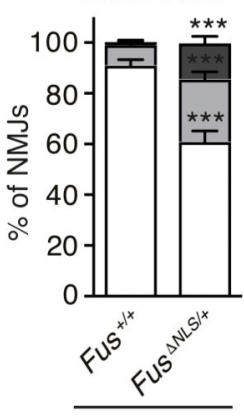

1 month
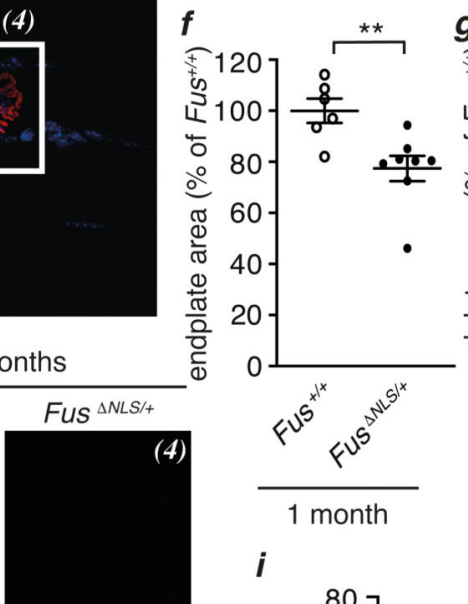

$$
i^{1}
$$

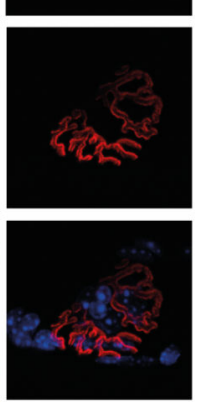

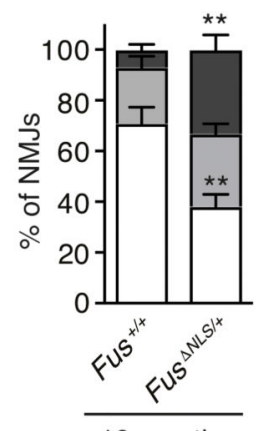
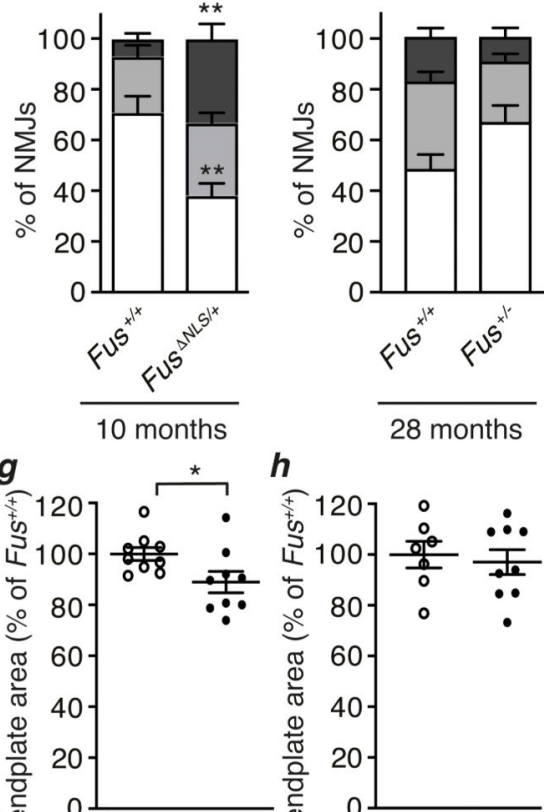

h
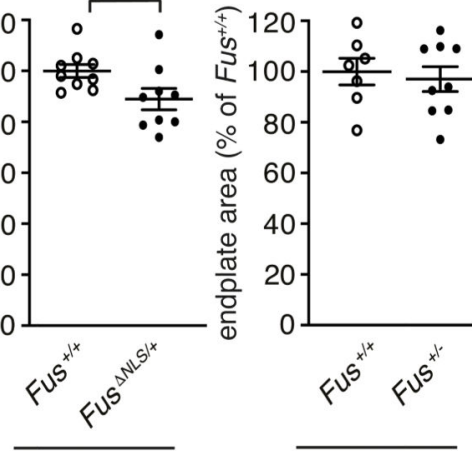

10 months

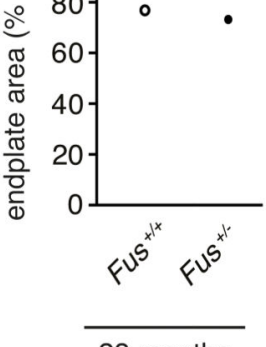

28 months

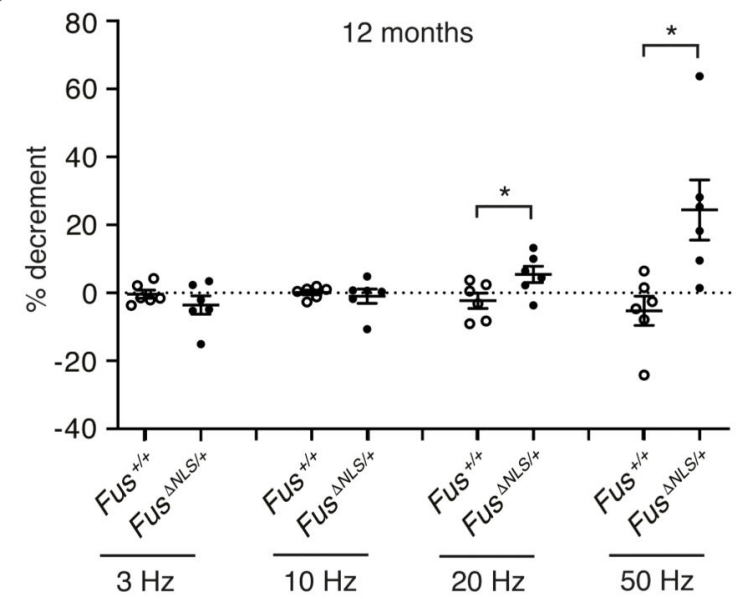

Figure 3. Neuromuscular junction (NMJ) defects in heterozygous $F u s^{\Delta N L S /+}$ mice. a, b, Representative images showing NMJs in TA muscles of 1-month-old and 10-month-old $\mathrm{Fus}^{+/+}$versus Fus ${ }^{\Delta \mathrm{NLS} /+}$ mice. Panel b shows magnifications of individual NMJs (1 to 4) delineated in panel a. Axons and presynaptic terminals were visualized by immunostaining with antibodies against neurofilament and synaptophysin (green). AChRs in muscle endplates were visualized using fluorescently labeled BTX (red). Nuclei were labeled with DAPI (blue). Scale bars: $50 \mu \mathrm{m}$ (a), $15 \mu \mathrm{m}$ (b). c-e, Bar graphs showing the percentage of innervated, partially denervated and fully denervated endplates in the TA muscle of 1- 
month-old (c) and 10-month-old (d) male Fus ${ }^{+/+}$versus Fus ${ }^{4 N L S /+}$ mice, and 28-month-old female $\mathrm{Fus}^{+/+}$versus Fus ${ }^{+/-}$mice (e). Two-tailed unpaired t-test; **p $<0.005$, ***p $<0.001$; $\mathrm{N}=6$ versus 8 (c); 6 versus 7 (d); 7 versus 9 mice (e), with $\sim 150$ NMJs analyzed per mouse. Average \pm SEM. f-h, Average endplate area (as \% of Fus ${ }^{+/+}$) in the TA muscle of 1-monthold (f) and 10-month-old (g) male Fus ${ }^{+/+}$(open circles) versus Fus ${ }^{4 N L S /+}$ (closed circles) mice, and 28-month-old female Fus ${ }^{+/+}$(open circles) versus Fus ${ }^{+/}$(closed circles) mice (h). Two-tailed unpaired t-test; * $\mathrm{p}=0.039, * * \mathrm{p}=0.0077 ; \mathrm{N}=6$ versus 8 (f); 9 versus 9 (g); 7 versus 9 mice (h), with $\sim 150$ NMJs analyzed per mouse. Average \pm SEM. i, Percentage decrement in maximal CMAP amplitude between the first and tenth stimulus administered at frequencies of 3,10, 20 or $50 \mathrm{~Hz}$. CMAP amplitude was recorded in gastrocnemius in response to repetitive stimulation (10x) of the sciatic nerve of 12-month-old female $\mathrm{Fus}^{+/+}$ (open circles) versus FuS ${ }^{\Delta N L S /+}$ (closed circles) mice. Two-tailed unpaired t-test; ${ }^{*} \mathrm{p}<0.05$; $\mathrm{N}=6$ versus 6 mice. Average \pm SEM. 


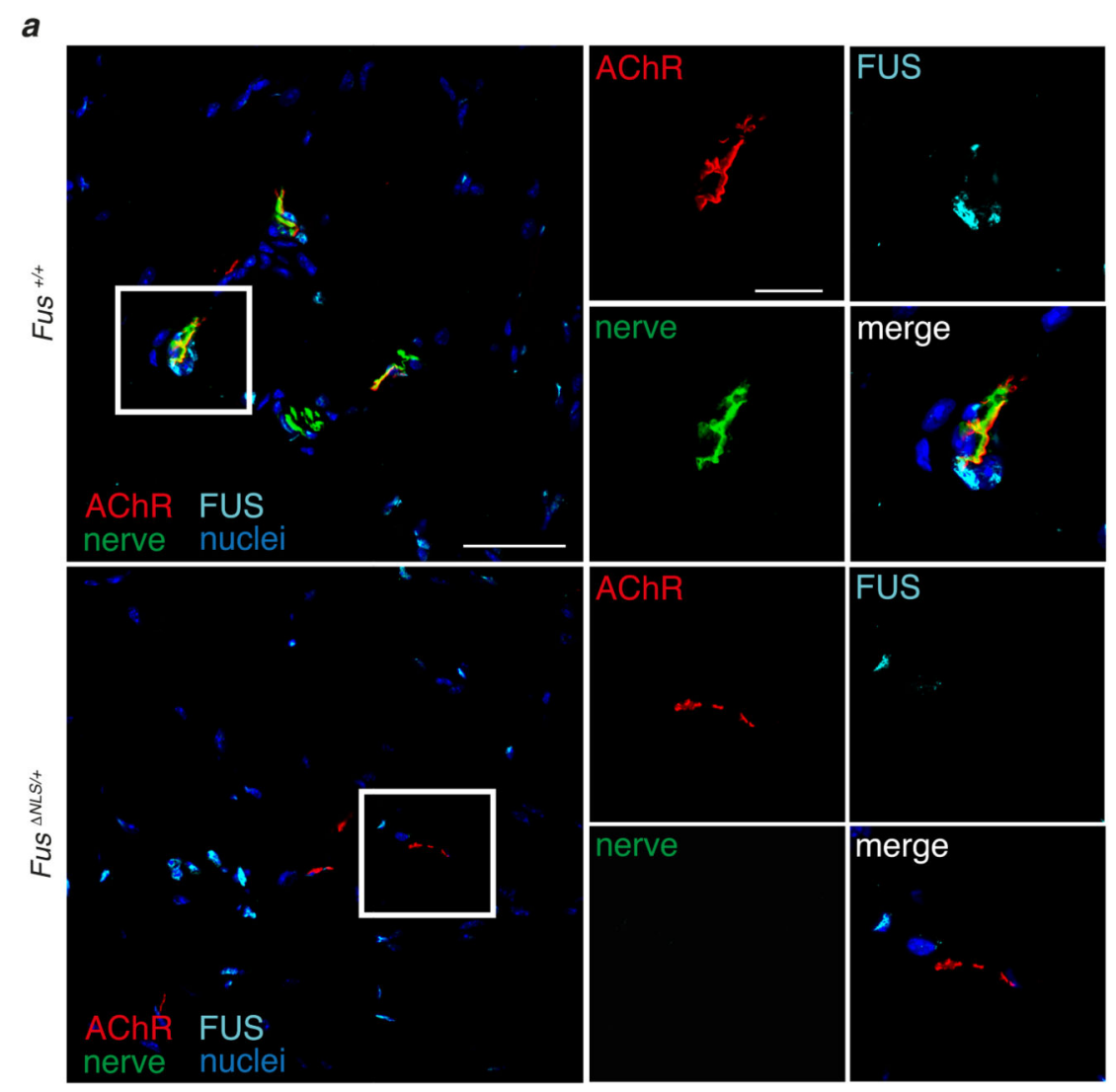

$c$

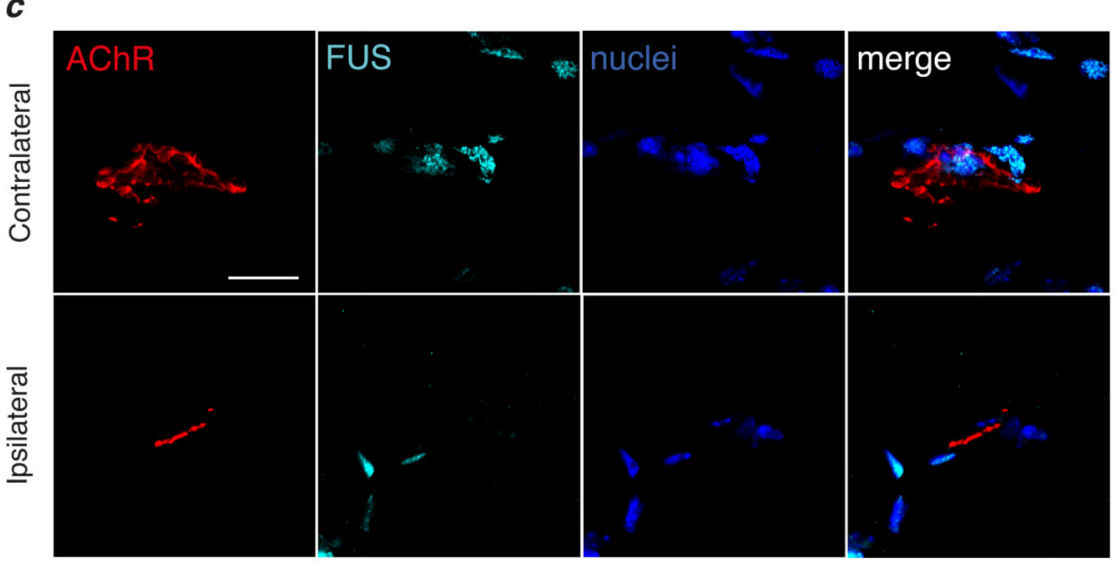

d

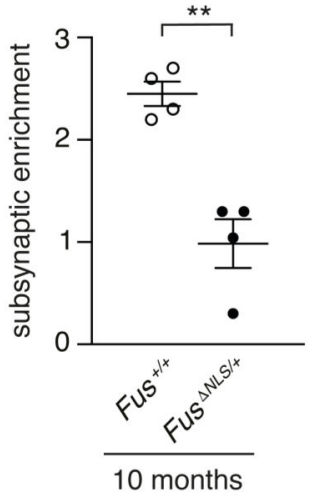

AChR FUS

nerve nuclei

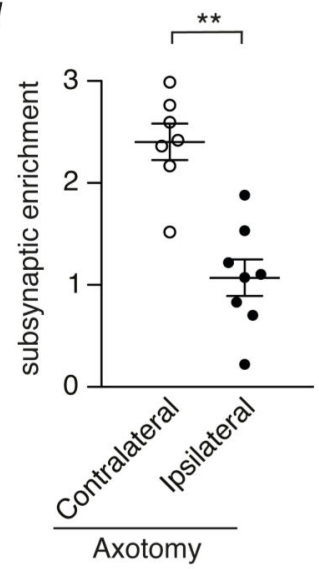

Figure 4. Innervation-dependent enrichment of FUS in subsynaptic nuclei.

a, Representative images showing FUS immunoreactivity (cyan) in the gastrocnemius muscle of 10 -month-old Fus ${ }^{+/+}$versus Fus ${ }^{\Delta N L S /+}$ mice. Axons and presynaptic terminals were visualized by immunostaining of neurofilament and synaptophysin (green). AChRs in muscle endplates were visualized using fluorescently labeled BTX (red). Scale bars: 50 $\mu \mathrm{m}$

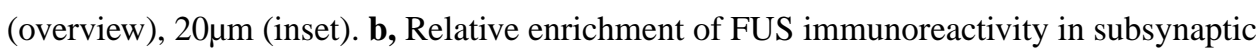
versus nonsynaptic nuclei in gastrocnemius muscle of 10-month-old male $\mathrm{Fus}^{+/+}$(open circles) versus Fus ${ }^{\Delta N L S /+}$ (closed circles) mice. Subsynaptic nuclei were defined as nuclei 
apposed to BTX-positive endplates. Two-tailed unpaired $\mathrm{t}$-test; ${ }^{* *} \mathrm{p}=0.0013 ; \mathrm{N}=4$ versus 4 mice. Average \pm SEM. c, Representative images showing FUS immunoreactivity (cyan) in the contralateral (upper panels) or ipsilateral (lower panels) gastrocnemius muscle of adult male wild type mice, 5 days after sciatic nerve axotomy. Scale bar: $20 \mu \mathrm{m}$. d, Quantification of subsynaptic enrichment of FUS in ipsilateral (closed circles) and contralateral (open circles) gastrocnemius. Two-tailed unpaired t-test with Welch's correction; $* * \mathrm{p}=0.0055 ; \mathrm{N}=$ 8 mice. Average \pm SEM. 

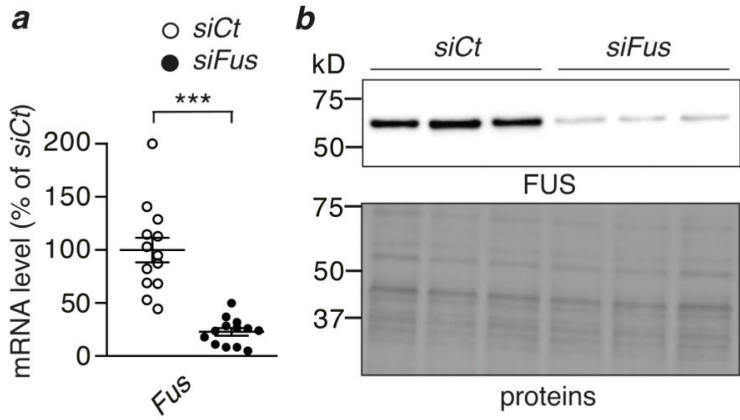

c o siCt
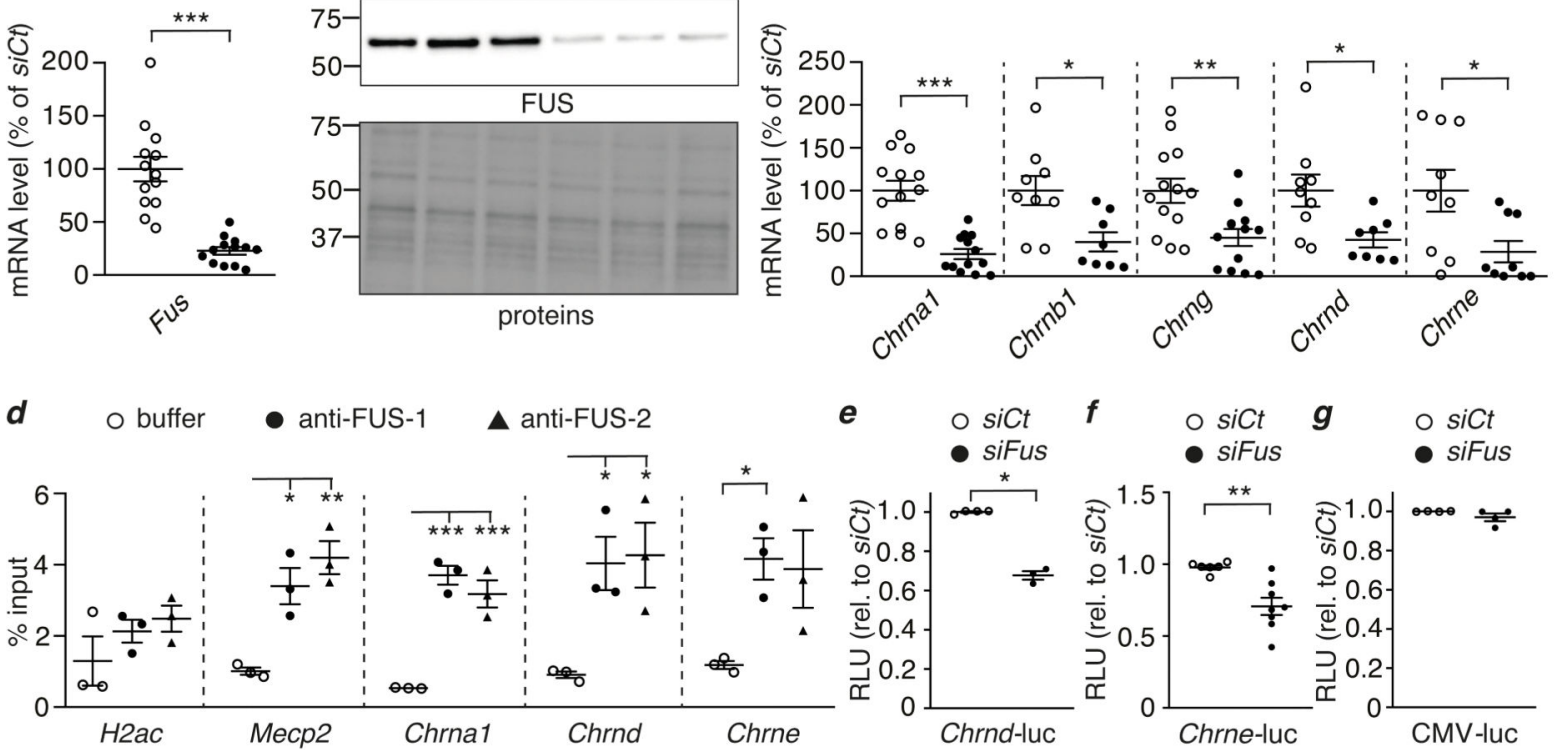

e

siCt

siFus

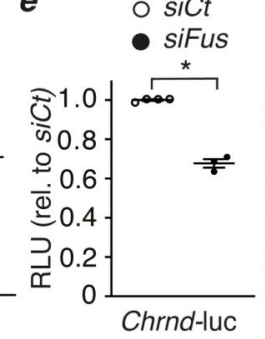

O siCt

- siFus

g $\quad$ siCt

Chrne-luc

CMV-luc

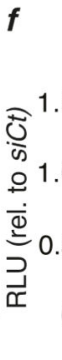

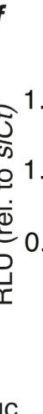

$\boldsymbol{h}$ O empty $\bullet$ hFUS_WT $\Delta$ hFUS_R495X $\boldsymbol{i}$

i
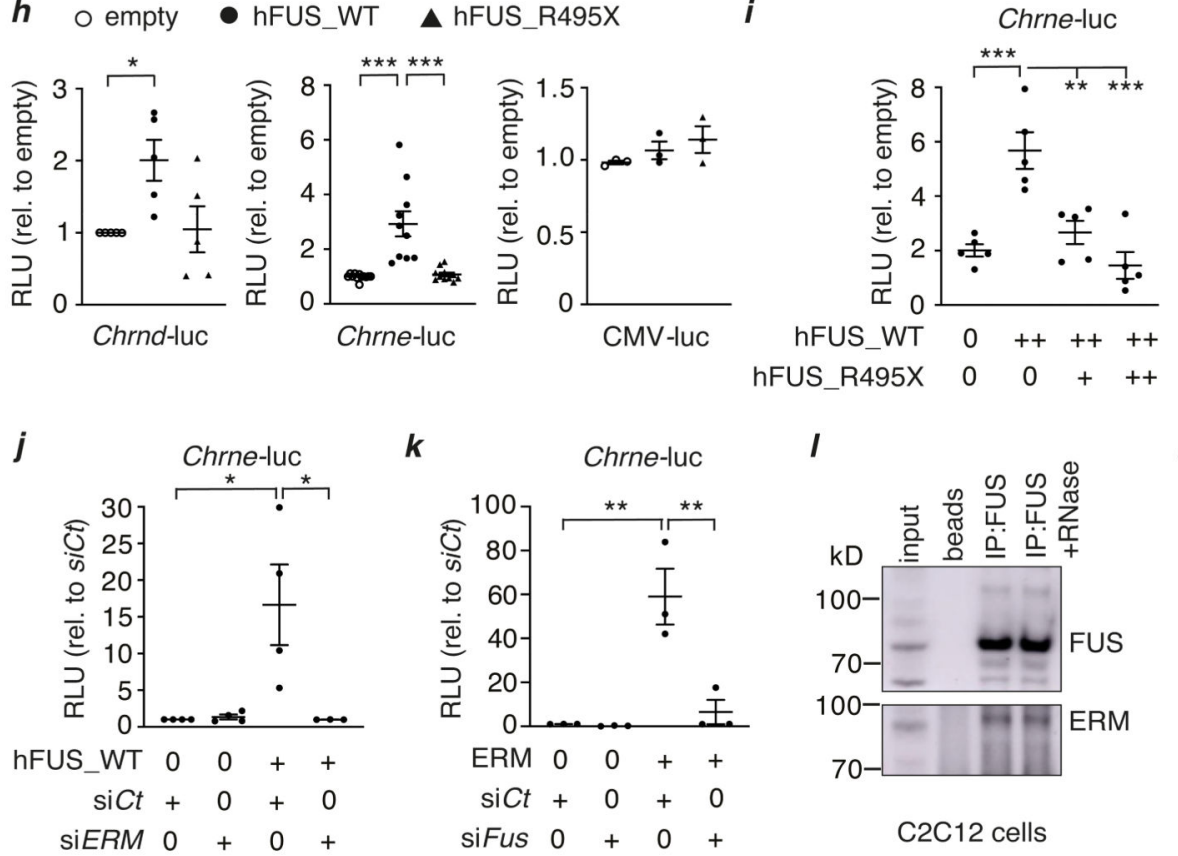

CMV-luc

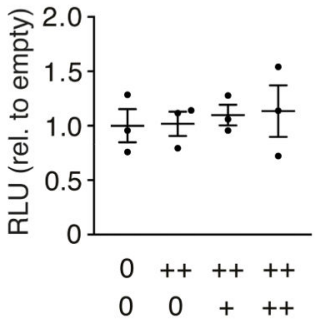

m

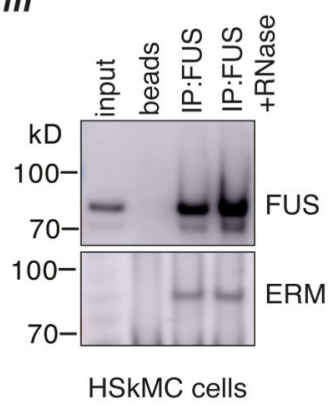

Figure 5. FUS regulates subsynaptic expression of $\mathrm{Chrn}$ genes.

a, b, Fus relative mRNA (a) and protein (b) levels in C2C12 cells $48 \mathrm{~h}$ after transfection with control siRNA ( siCt, $100 \mathrm{nM}$ ) or siRNA targeting Fus (siFus, $100 \mathrm{nM}$ ). In b, lower panel: total protein stain of the corresponding western blot. (a) Two-tailed unpaired t-test with Welch's correction; ***p<0.0001; $\mathrm{N}=3$ independent transfections with 4-5 technical replicates per transfection. Average \pm SEM. c, Relative mRNA levels of Chrn genes in C2C12 cells processed as in a. Two-tailed unpaired t-test (with Welch's correction for Chrnal and Chrnd); ***p<0.0001, **p=0.0046, *p<0.05; N=3 independent transfections 
with 4-5 technical replicates per transfection. Average \pm SEM. d, Fraction of chromatin immunoprecipitated (\% input) with buffer, or with two different anti-FUS antibodies (antiFUS-1 and anti-FUS-2) as detected using qPCR with primers located in the promoter regions of corresponding genes. One-way ANOVA with Dunnett's multiple comparisons test; $* * * \mathrm{p}<0.001,{ }^{*} \mathrm{p}=0.0025,{ }^{*} \mathrm{p}<0.05 ; \mathrm{N}=3$ independent samples per condition. Average \pm SEM. e-g, Light units (RLU) relative to siCt in $\mathrm{C} 2 \mathrm{C} 12$ cells $24 \mathrm{~h}$ after transfection with siCt ( $25 \mathrm{nM}$ ) or siFus (25 nM) and luciferase reporter plasmids carrying promoters of Chrnd (e), Chrne (f) genes or CMV (g). Two-tailed nested t-test; ** $\mathrm{p}=0.0014,{ }^{*} \mathrm{p}=0.049 ; \mathrm{N}=4$ (e, g) and 6 (siCt) or 8 (siFus) (f) independent transfections, each consisting of 6 technical replicates. Average \pm SEM. h, Light units relative to the empty control in $\mathrm{C} 2 \mathrm{C} 12$ cells transfected with the same reporter plasmids and plasmids expressing hFUS_WT, hFUS_R495X or empty control plasmid. Nested one-way ANOVA with Tukey's multiple comparisons test; ***p<0.0005, *p=0.025; $\mathrm{N}=5$ (Chrnd-luc), 12 (Chrne-luc), 3 (CMV-luc) independent transfections, each representing the average of 4 ( 8 for $C M V$-luc) technical replicates. Average \pm SEM. i, Light units relative to empty control plasmid in $\mathrm{C} 2 \mathrm{C} 12$ cells transfected with indicated reporter plasmids, hFUS_WT expression plasmid and increasing doses of hFUS_R495X. Nested one-way ANOVA with Tukey's multiple comparisons test; *** $<<0.0005, * * \mathrm{p}=0.0022 ; \mathrm{N}=5$ (Chrne-luc), 3 (CMV-luc) independent transfections, each consisting of 5 (Chrne-luc) or 4 (CMV-luc) technical replicates. Average \pm SEM. j, k, Light units relative to siCt in $\mathrm{C} 2 \mathrm{C} 12$ cells $24 \mathrm{~h}$ after transfection of Chrne-luciferase plasmid and either siCt $(25 \mathrm{nM})$ or siErm $(25 \mathrm{nM}, \mathrm{j})$ or siFus $(25 \mathrm{nM}, \mathrm{k})$, and an expression plasmid for either hFUS_WT (j) or ERM (k) or empty control. Nested one-way ANOVA with Tukey's multiple comparisons test $* * \mathrm{p}<0.005,{ }^{*} \mathrm{p}<0.05 ; \mathrm{N}=4(j), 3(k)$ independent transfections, each consisting of $6(j)$ or $4(k)$ technical replicates. Average \pm SEM. $1, \mathbf{m}$, Endogenous FUS was immunoprecipitated from $\mathrm{C} 2 \mathrm{C} 12$ (l) and HSkMC $(m)$ cells using a specific antibody (IP) or beads only, in the presence or absence of RNase. Native lysates (input) and immunoprecipitates were analyzed by Western blotting with anti-FUS or anti-ERM antibodies. Co-IP experiments were independently repeated $3(I)$ or $4(m)$ times with similar results. Blot images in panels b, 1 and $\mathrm{m}$ were cropped. Supplementary Figure 11 shows uncropped blots. 


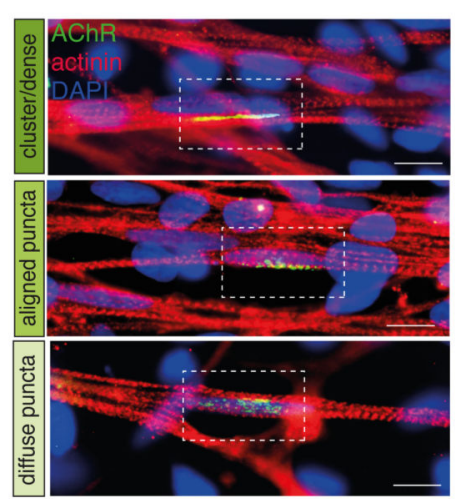

c
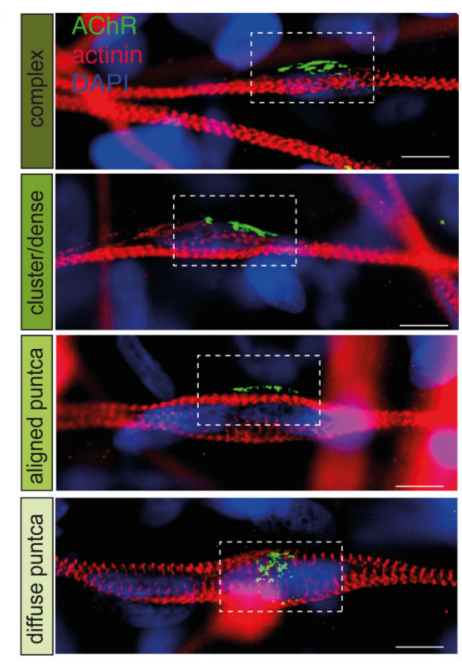

e

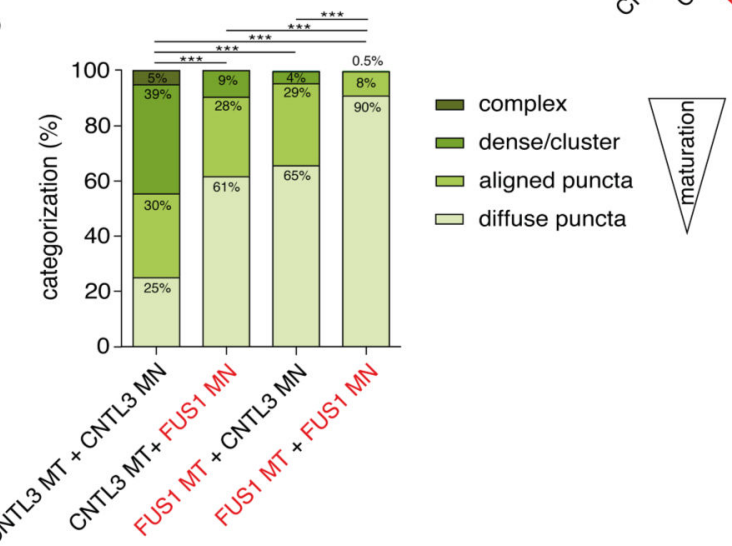

b

$$
\begin{aligned}
& \square \text { dense/cluster } \\
& \square \text { aligned puncta } \\
& \square \text { diffuse puncta }
\end{aligned}
$$

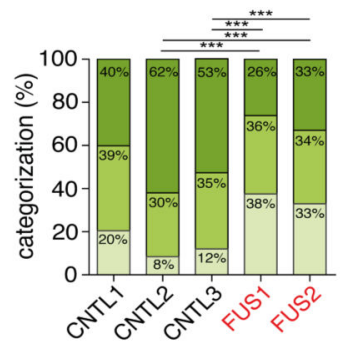

d $\quad$ complex

$\square$ dense/cluste

$\square$ aligned puncta

$\square$ diffuse puncta
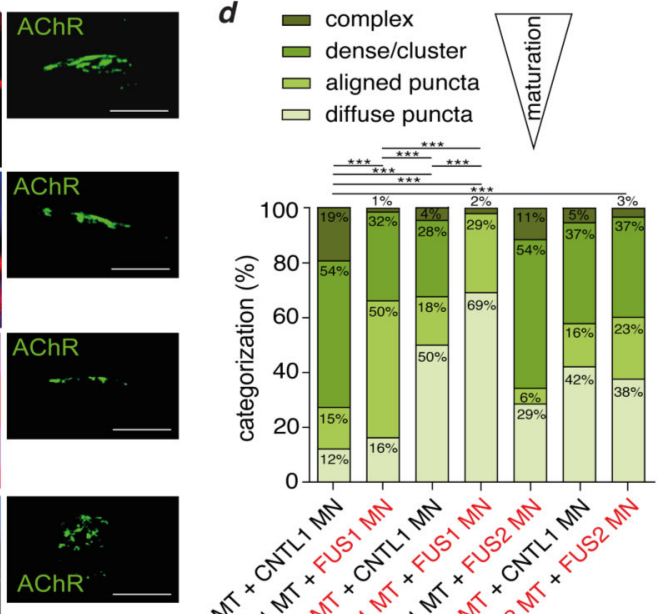

Figure 6. Impaired endplate maturation in iPSC-derived myotubes from FUS-ALS patients. a, Structure of AChR clusters on hiPSC-derived myotubes was visualized with BTX (green) and assorted based on their morphology in three categories with increasing maturity (diffuse puncta, aligned puncta, dense/cluster). Myotubes were immunolabeled with actinin (red) and nuclei with DAPI (blue). Scale bar: $10 \mu \mathrm{m}$. b, Quantification of maturation state revealed less mature endplate structures in both patient-derived cell lines compared to CNTL2 and CNTL3. Two-sided Fisher's Exact Test with Bonferroni correction for multiple testing; $* * * \mathrm{p}<0.0005 ; \mathrm{N}$ (number of endplates)= 127 (CNTL1), 116 (CNTL2), 71 (CNTL3), 88 
(FUS1), 94 (FUS2). N= 3 independent cultures per condition. $\mathbf{c}$, Structure of AChR clusters in hiPSC-derived motor neuron-myotube co-cultures was visualized with BTX (green) and assorted based on their morphology in four categories with increasing maturity (diffuse puncta, aligned puncta, dense/cluster or complex). Myotubes were immunolabeled with actinin (red) and nuclei with DAPI (blue). Scale bar: 10 $\mu \mathrm{m}$. d, Quantification of maturation state is shown in the bar graph, revealing less mature endplate-like structures in co-cultures in which myotubes and/or motor neurons were derived from FUS-ALS patients. Two-sided Fisher's Exact Test with Bonferroni correction for multiple testing; ***p<0.00005; N (number of endplates) $=99($ CNTL1 MT + CNTL 1 MN), $74($ CNTL1 MT + FUS1 MN), 226 (FUS1 MT + CNTL1 MN), 52 (FUS1 MT + FUS1 MN), 35 (CNTL1 MT + FUS2 MN), 19 (FUS2 MT + CNTL1 MN), 93 (FUS2 MT + FUS2 MT). N= 3 independent co-cultures per condition. e, Quantification of maturation state in motor-neuron myotube co-cultures derived from FUS1 and its isogenic CNTL3. Two-sided Fisher's Exact Test with Bonferroni correction for multiple testing; $* * * \mathrm{p}<5 \times 10^{-8} ; \mathrm{N}$ (number of endplates) $=273$ (CNTL3 MT + CNTL3 MN), 73 (CNTL3 MT + FUS1 MN), 381 (FUS1 MN + CNTL3 MT), 285 (FUS1 MT + FUS1 MN). N= 3 independent co-cultures per condition. 
a

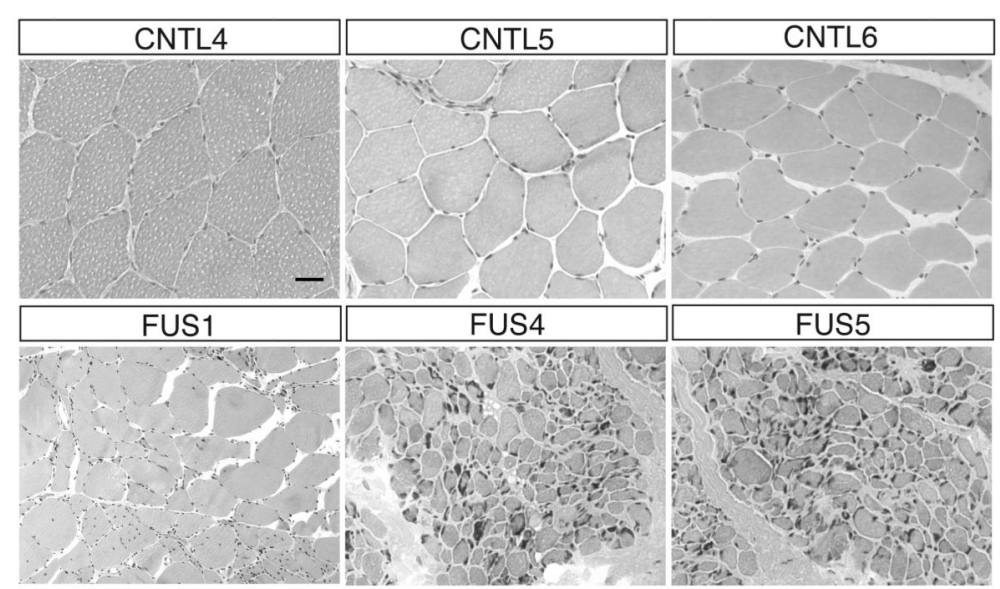

b

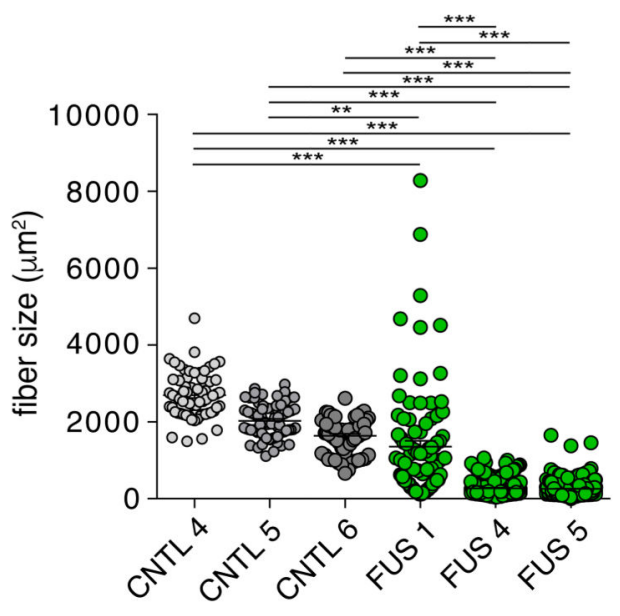

C

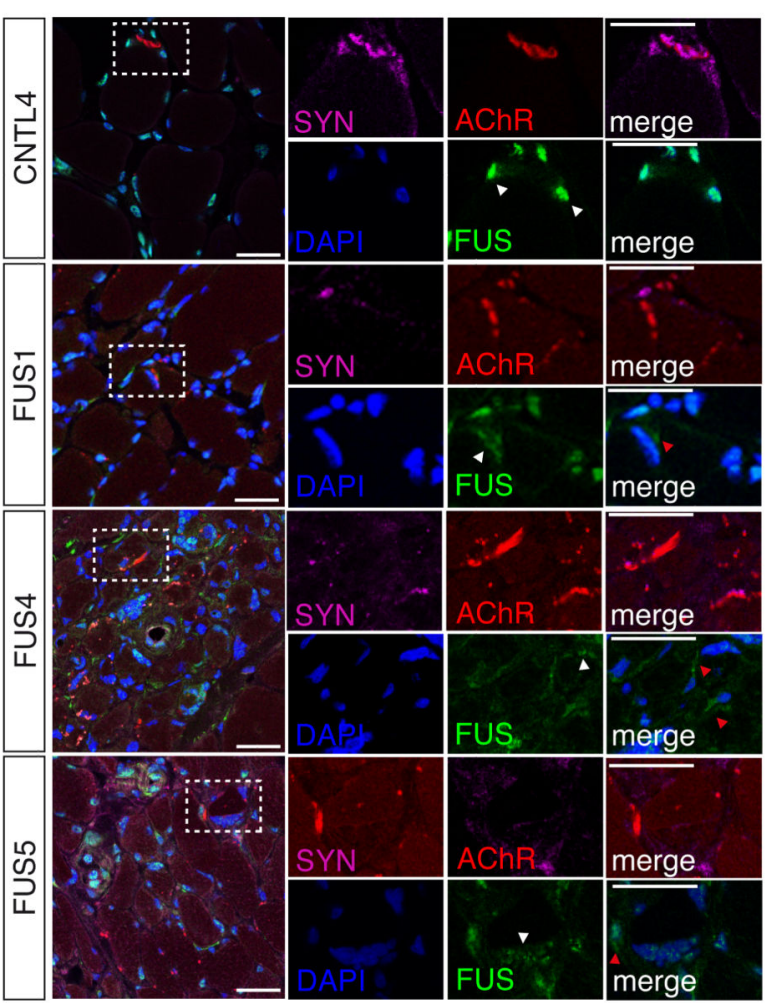

d

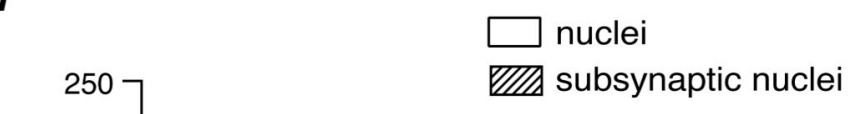

Figure 7. Skeletal muscle of FUS-ALS patients displays muscle atrophy, FUS mislocalization, and impaired FUS enrichment in subsynaptic nuclei.

a, H\&E staining on muscle biopsies revealed muscle atrophy in FUS-ALS patients, with angular and shrunken myofibers, increased density of nuclei and connective tissue. Scale bar: $50 \mu \mathrm{m}$. b, Quantification of muscle fiber size in muscle biopsies from control (gray circles) and FUS-ALS patients (green circles). **p=0.0015; ***p<0.0001 by Kruskal-Wallis test with Dunn's multiple comparisons test. Number of muscle fibers $=66$ (CNTL4), 72 (CNTL5), 73 (CNTL6), 91 (FUS1), 349 (FUS4), 415 (FUS5). Average \pm SEM. c, Staining of skeletal muscle biopsies for FUS (green), synaptophysin (SYN, magenta, labels presynaptic terminals), AChR (BTX, red) and DAPI (blue). White arrowheads indicate FUS 
localization in the nucleus, which was diffuse in CNTL biopsies, whereas in FUS-ALS patients staining was often either lost, reduced, or showed a granular pattern. Red arrowheads indicate cytoplasmic FUS localization in FUS-ALS patients. Scale bar: $20 \mu \mathrm{m}$. d, Box-Whisker plot showing the quantification of FUS staining intensity in either all nuclei or selectively in subsynaptic nuclei. Two-tailed Mann-Whitney U; *p $<0.05$, *** $<<0.0001 ; \mathrm{N}$ (all nuclei-subsynaptic nuclei) $=218-8$ (CNTL4), 416-16 (CNTL5), 232-12 (CNTL6), 493-12 (FUS1), 910-11 (FUS4), 3397-39 (FUS5). Median with 25 and 75 percentile (box) and minima and maxima (whiskers) are shown. 